\title{
Spaces of generalized smoothness on $h$-sets and related Dirichlet forms
}

\author{
by \\ V. Knopova and M. ZäHLE (Jena)
}

\begin{abstract}
The paper is devoted to spaces of generalized smoothness on so-called $h$-sets. First we find quarkonial representations of isotropic spaces of generalized smoothness on $\mathbb{R}^{n}$ and on an $h$-set. Then we investigate representations of such spaces via differences, which are very helpful when we want to find an explicit representation of the domain of a Dirichlet form on $h$-sets. We prove that both representations are equivalent, and also find the domain of some time-changed Dirichlet form on an $h$-set.
\end{abstract}

1. Introduction. The paper is devoted to Besov-type spaces on $h$-sets. Such sets were defined and studied in Edmunds and Triebel [5], [6]; see also Bricchi [3] and [4]. Our goal is to study more general spaces on such sets, and show how these results can be applied when we look for the domain of Dirichlet forms on an $h$-set, associated with restrictions of certain symmetric Lévy processes to this set.

Let $h:(0,1] \rightarrow \mathbb{R}$ be a given continuous positive non-decreasing function. Such functions are sometimes called gauge functions. A compact set $\Gamma$ is called an $h$-set if there exists a finite Radon measure $\mu$ such that

(1) $\operatorname{supp} \mu=\Gamma$,

(2) $c_{1} h(r) \leq \mu(B(\gamma, r)) \leq c_{2} h(r), \gamma \in \Gamma, 0<r \leq 1$,

where $c_{1}$ and $c_{2}$ are positive constants, and $B(\gamma, r)$ is the ball centered at $\gamma$ with radius $r$. Then $\mu$ is called an $h$-measure, and the gauge function, for which there exists an $h$-set, is called the measure function. For example, the

2000 Mathematics Subject Classification: 46E35, 42B35, 28A80, 60J75.

Key words and phrases: space of generalized smoothness, quarkonial representation, $h$-sets, Dirichlet form, time-changed Dirichlet form.

The first-named author gratefully acknowledges the DFG grant from the University of Jena. 
functions

$$
\begin{aligned}
& h(r)=r^{d}, \quad 0 \leq d \leq n, \\
& h(r)=r^{d}|\log r|^{b}, \quad 0<d<n, b \in \mathbb{R}, \\
& h(r)=r^{d} \exp \left[b|\log r|^{\kappa}\right], \quad 0 \leq d \leq n, b \in \mathbb{R}, 0<\kappa<1,
\end{aligned}
$$

are measure functions (in order to have gauge functions, the last two examples are defined for small $r$, and then extended to $(0,1])$. For more examples see [4].

For $h(r)=r^{d}$ such $\Gamma$ are called $d$-sets (see Jonsson [19], Jonsson and Wallin [20], and Triebel [30], [31]), and these are in turn a generalization of self-similar sets with the open set condition. For representation of norms in spaces of Lipschitz type on metric measure spaces cf. also [32].

In Section 3.2 we assume that $h$ satisfies the following condition: there exist $0<d \leq s \leq n$ such that

$$
c_{1} \lambda^{s}<\frac{h(\lambda t)}{h(t)}<c_{2} \lambda^{d}
$$

for all $0<t, \lambda<1$.

We are interested in the representation of some isotropic spaces of generalized smoothness on $\mathbb{R}^{n}$ via differences. Similar questions were considered in Haroske and Moura [13] and Moura [25]; see also Triebel [29] for ordinary Besov and Triebel-Lizorkin spaces. Such an approach is very helpful when we need to obtain an explicit representation of the domain of some Dirichlet forms, and also when we need to find explicitly the trace space on a set of lower dimension. In such a way the traces of some classical Besov spaces on $d$-sets were constructed in [20], and on a generalized version of $h$-sets in [19], and it was proved that there exists a continuous restriction to such trace spaces, with corresponding continuous extension. The methods used in [19] and [20] are not directly applicable in our situation, since in our case the kernel in the representation of the Dirichlet form considered is more general than the Bessel kernel, and it is harder to get estimates for it. In order to employ Tauberian-type theorems for so-called extended regularly varying functions (see [2]) for this problem, we consider kernels of some special type (see below).

Another approach is to obtain the representation of spaces of generalized smoothness on $h$-sets using quarkonial decompositions; see [30] and [31]. To find such decompositions we use the methods developed in [30] and [31], as well as in [3]. The advantage of this approach is that we can obtain the representation of the trace of a function $u$ on $\Gamma$ just by index shifting in the quarkonial representation of $u$ on $\mathbb{R}^{n}$. While it is easier to find the representation of the trace space using quarkonial decompositions, the representation via differences is very helpful when we want to study Dirichlet 
forms on $\Gamma$. In Section 3.3 we will show that both methods of tracing give the same trace space.

In Section 2 we define generalized quarks on $\mathbb{R}^{n}$ and on an $h$-set. Then we find the quarkonial representation of the Besov-type space of generalized smoothness $B_{p q}^{\sigma, N}\left(\mathbb{R}^{n}\right)$ on $\mathbb{R}^{n}$. Here $\sigma=\left(\sigma_{j}\right)_{j \geq 0}$ and $N=\left(N_{j}\right)_{j \geq 0}$ are respectively an admissible sequence and a strongly increasing sequence (see Definition 1 below). We show that such a definition of a space of generalized smoothness is equivalent to that via atoms. Then we prove the trace theorem, i.e. under some general condition on the sequences $\sigma$ and $N$, the Bernstein function $f$ and the gauge function $h$ we find the representation of the trace space on an $h$-set, and show that there exist continuous restriction and extension.

In Section 3 we find the representation of the Triebel-Lizorkin-type space of generalized smoothness $F_{p q}^{\sigma, N}\left(\mathbb{R}^{n}\right)$ via differences. Here the sequence $N=$ $\left(N_{j}\right)_{j \geq 0}$ is defined in the following way. Let $f$ be a Bernstein function, i.e. $f \in \bar{C}^{\infty}(0, \infty), f \geq 0$, and $(-1)^{n} f^{(n)} \leq 0$ for all $n \geq 1$. In our paper we assume that there exists $\kappa \in[1, \infty)$ such that

$$
f(t) t^{-1 / \kappa} \text { is increasing as } t \rightarrow \infty \text {. }
$$

Then put $N_{j}=\sqrt{f^{-1}\left(2^{2 j}\right)}, j \geq 0$. For such $N_{j}$ the inequality

$$
N_{j+1} \leq 2^{\kappa} N_{j}
$$

holds (see [9]). This inequality will play a significant role when we look for the trace space of some space of generalized smoothness on $\Gamma$.

Starting with a representation of $B_{p p}^{\sigma, N}\left(\mathbb{R}^{n}\right)\left(=F_{p p}^{\sigma, N}\left(\mathbb{R}^{n}\right)\right)$ via differences, we derive another representation of the trace of $B_{p p}^{\sigma, N}\left(\mathbb{R}^{n}\right)$ on an $h$-set. In the end we prove that the method used in [19] (or [20]) gives in our case the same result as the method described in Section 2. We put the detailed proofs in Appendices II and III.

In Section 3.2 we will need an additional assumption on a Bernstein function $f$, i.e. we assume that

$$
\frac{1}{c^{\delta}} \leq \frac{f(c \lambda)}{f(\lambda)} \leq c \quad \text { for all } \lambda>0, c \geq 1, \text { and some } 0<\delta<1
$$

This condition is sufficient for the proof of the continuity of the restriction by Jonsson and Wallin's method.

For example, condition (4) is satisfied for Bernstein functions $f(\lambda)=\lambda^{\alpha}$, $f(\lambda)=\lambda^{\alpha} \ln \left(1+\lambda^{\alpha}\right), \lambda>0,0<\alpha<1$. Note that for $\delta=1$ equation (4) holds for any Bernstein function (see [15]).

Dirichlet forms and related stable-like jump processes on $d$-sets were studied in [26], [21], [22], [12], etc. In Section 4 we give the representation of a Dirichlet form which is equivalent to the Dirichlet form $(\mathcal{E}, D(\mathcal{E}))$ associated with a symmetric Lévy process with exponent $f\left(|\xi|^{2}\right)$, where $f$ is a Bernstein 
function. Applying the results from Sections 2 and 3, we find the domain of the trace of the Dirichlet form $(\mathcal{E}, D(\mathcal{E}))$ on an $h$-set. For the general theory of Dirichlet forms see, for example, [11]. Here we only indicate that if $\psi$ is a continuous negative-definite function, then

$$
\mathcal{E}(u, v)=\int_{\mathbb{R}^{n}} \psi(\xi) \widehat{u}(\xi) \widehat{v}(\xi) d \xi
$$

is the Dirichlet form associated with $\psi$. Then $D(\mathcal{E})=H_{2}^{\psi, 1}\left(\mathbb{R}^{n}\right)$, where $H_{p}^{\psi, s}\left(\mathbb{R}^{n}\right)$ is the $\psi$-Bessel potential space of order $s$ (see [16] and [8]). We are especially interested in the case when

$$
\psi(\xi)=f\left(|\xi|^{2}\right),
$$

where $f$ is a Bernstein function.

Since $H_{p}^{f\left(|\cdot|^{2}\right), s}\left(\mathbb{R}^{n}\right)=F_{p 2}^{\sigma, N}\left(\mathbb{R}^{n}\right)$, where $\sigma=\left(2^{j s}\right)_{j \geq 0}$, and $N=\left(N_{j}\right)_{j \geq 0}$, $N_{j}=\sqrt{f^{-1}\left(2^{2 j}\right)}$ (see [9] for the proof), we can apply the tools from the theory of function spaces developed in Sections 2 and 3 to get the representation of the domain of the time-changed Dirichlet form $(\check{\mathcal{E}}, D(\check{\mathcal{E}}))$, which corresponds to a Markov process on an $h$-set. This domain is just the trace of $H^{f\left(|\cdot|^{2}\right), 1}\left(\mathbb{R}^{n}\right)=D(\mathcal{E})$ on this $h$-set. Therefore the domain of the time-changed Dirichlet form $(\check{\mathcal{E}}, D(\check{\mathcal{E}}))$ is

$$
\begin{aligned}
D(\check{\mathcal{E}})= & \left\{u \in L_{2}(\Gamma):\right. \\
& \left.\iint_{|x-y|<1} \frac{f\left(|x-y|^{-2}\right)}{|x-y|^{-n} h(|x-y|)^{2}}|u(x)-u(y)|^{2} \mu(d x) \mu(d y)<\infty\right\} .
\end{aligned}
$$

\section{Quarkonial representations. Trace theorem I}

2.1. Quarkonial representations on $\mathbb{R}^{n}$. This section is devoted to the quarkonial representation of spaces of generalized smoothness on $\mathbb{R}^{n}$ and on an $h$-set $\Gamma$. We will prove some technical results, which allow us to find the trace space of a Besov-type space of generalized smoothness (see Definition 3 below) on an $h$-set.

Notation. $\widehat{u}$ is the Fourier transform

$$
\widehat{u}(\xi)=\int_{\mathbb{R}^{n}} e^{-i \xi x} u(x) d x,
$$

and $(u(\cdot))^{\vee}$ is the inverse Fourier transform. $S\left(\mathbb{R}^{n}\right)$ and $S^{\prime}\left(\mathbb{R}^{n}\right)$ are the Schwartz space and the dual Schwartz space respectively.

We will use several definitions of spaces of generalized smoothness, which in the end appear to be equivalent. To give the first definition, we need admissible and strongly increasing sequences, and the related decomposition of unity. We will follow the presentation given in [9] (see also [7]). 
Definition 1. A sequence $\gamma=\left(\gamma_{j}\right)_{j \in \mathbb{N}_{0}}$ of positive real numbers is called

(i) almost increasing if there exists $d_{0}>0$ such that $d_{0} \gamma_{j} \leq \gamma_{k}$ for all $j, k$ such that $0 \leq j \leq k$;

(ii) strongly increasing if it is almost increasing, and in addition there exists a natural number $\kappa_{0}$ such that

$$
2 \gamma_{j} \leq \gamma_{k} \quad \text { for all } j \text { and } k \text { and } j+\kappa_{0} \leq k ;
$$

(iii) of bounded growth if there are positive constants $d_{1}$ and $J_{0} \in \mathbb{N}_{0}$ such that

$$
\gamma_{j+1} \leq d_{1} \gamma_{j} \text { for all } j \geq J_{0} .
$$

Let $\left(\sigma_{j}\right)_{j \in \mathbb{N}_{0}}$ be a sequence which satisfies for some $d_{0}, d_{1}>0$ the inequality

$$
d_{0} \sigma_{j} \leq \sigma_{j+1} \leq d_{1} \sigma_{j} \quad \text { for all } j \in \mathbb{N} .
$$

This means that both $\left(\sigma_{j}\right)_{j \in \mathbb{N}_{0}}$ and $\left(\sigma_{j}^{-1}\right)_{j \in \mathbb{N}_{0}}$ are of bounded growth. We will call the sequences which satisfy (5) admissible.

For simplicity we will assume below that $\kappa_{0}=J_{0}=1$.

Definition 2. Let $\left(N_{k}\right)_{k \geq 0}$ be a strongly increasing sequence. Define

$$
\begin{aligned}
& \Omega_{0}^{N}=\left\{\xi \in \mathbb{R}^{n}:|\xi| \leq N_{0}\right\}, \\
& \Omega_{j}^{N}=\left\{\xi \in \mathbb{R}^{n}: N_{j-1} \leq|\xi| \leq N_{j+1}\right\}, \quad j=1,2, \ldots .
\end{aligned}
$$

Let $\Phi^{N}$ be the collection of all function systems $\left(\varphi_{j}^{N}\right)_{j \geq 0}$ such that $\varphi_{j}^{N} \in$ $C_{0}^{\infty}\left(\mathbb{R}^{n}\right), \varphi_{j}^{N}(\xi) \geq 0, \xi \in \mathbb{R}^{n}$, for any $j \geq 0, \operatorname{supp} \varphi_{j}^{N} \subset \Omega_{j}^{N}$, and

$$
\sum_{j \geq 0}^{\infty} \varphi_{j}^{N}(\xi)=1 \quad \text { for all } \xi \in \mathbb{R}^{n} .
$$

Definition 3. Let $N=\left(N_{j}\right)_{j \in \mathbb{N}_{0}}$ be a strongly increasing sequence, $\left(\varphi_{j}^{N}\right)_{j \geq 0} \in \Phi^{N}$, and $\left(\sigma_{j}\right)_{j \in \mathbb{N}_{0}}$ be an admissible sequence.

(i) Let $1<p<\infty, 1 \leq q \leq \infty$. Then the Besov space of generalized smoothness is

$$
\begin{aligned}
& B_{p q}^{\sigma, N}\left(\mathbb{R}^{n}\right)=\left\{g \in S^{\prime}\left(\mathbb{R}^{n}\right):\right. \\
&\left.\left\|g\left|B_{p q}^{\sigma, N}\left(\mathbb{R}^{n}\right)\|=\|\left(\sigma_{j} \varphi_{j}^{N}(D) g\right)_{j \in \mathbb{N}_{0}}\right| l_{q}\left(L_{p}\right)\right\|<\infty\right\} .
\end{aligned}
$$

(ii) Let $1<p<\infty, 1<q<\infty$. Then the Triebel-Lizorkin space of generalized smoothness is

$$
\begin{aligned}
F_{p q}^{\sigma, N}\left(\mathbb{R}^{n}\right)=\left\{g \in S^{\prime}\left(\mathbb{R}^{n}\right):\right. & \\
& \left.\left\|g\left|F_{p q}^{\sigma, N}\left(\mathbb{R}^{n}\right)\|=\|\left(\sigma_{j} \varphi_{j}^{N}(D) g\right)_{j \in \mathbb{N}_{0}}\right| L_{p}\left(l_{q}\right)\right\|<\infty\right\} .
\end{aligned}
$$

Here and in the following for $\varphi \in S\left(\mathbb{R}^{n}\right)$ (or $\varphi \in C_{0}^{\infty}\left(\mathbb{R}^{n}\right)$ ) we will understand $\varphi(D) u$ as $\varphi(D) u(x)=(\varphi(\cdot) \widehat{u})^{\vee}(x)$. 
Remark 4. For the classical Besov and Triebel-Lizorkin spaces $B_{p q}^{s}\left(\mathbb{R}^{n}\right)$ and $F_{p q}^{s}\left(\mathbb{R}^{n}\right)$ put $\sigma_{j}=2^{j s}$ and $N_{j}=2^{j}, j \geq 0$.

We need the following auxiliary sequence spaces: Let $k \in \mathbb{N}_{0}$, and let $1_{k m}(x)$ be the characteristic function on $Q_{k m}$. For $0<p \leq \infty$ put $1_{k m}^{(p)}(x)=$ $N_{k}^{n / p} 1_{k m}(x)$.

Definition 5. Let $0<p \leq \infty, 0<q \leq \infty$. Then:

(i) $b_{p q}$ is the collection of all sequences $\lambda=\left\{\lambda_{k m} \in \mathbb{C}: k \in \mathbb{N}_{0}, m \in \mathbb{Z}^{n}\right\}$ such that

$$
\left\|\lambda \mid b_{p q}\right\|=\left(\sum_{k=0}^{\infty}\left(\sum_{m \in \mathbb{Z}^{n}}\left|\lambda_{k m}\right|^{p}\right)^{q / p}\right)^{1 / q} .
$$

(ii) $f_{p q}^{N}$ is the collection of all sequences $\lambda=\left\{\lambda_{k m} \in \mathbb{C}: k \in \mathbb{N}_{0}, m \in \mathbb{Z}^{n}\right\}$ such that

$$
\left\|\lambda\left|f_{p q}^{N}\|=\|\left(\sum_{k=0}^{\infty} \sum_{m \in \mathbb{Z}^{n}}\left|\lambda_{k m} 1_{k m}^{(p)}(\cdot)\right|^{q}\right)^{1 / q}\right| L_{p}\left(\mathbb{R}^{n}\right)\right\| .
$$

Sometimes we will also need atomic representations of spaces of generalized smoothness.

Definition 6. Let $Q_{k m}$ be a cube in $\mathbb{R}^{n}$ centered at $N_{k}^{-1} m=\left(m_{1} / N_{k}, \ldots\right.$ $\left.\ldots, m_{n} / N_{k}\right)$, which has sides parallel to the axes and side length $1 / N_{k}$, and denote by $c Q_{k m}$ the cube concentric with $Q_{k m}$, with side length enlarged by the factor $c$. Let $\left(\sigma_{k}\right)_{k \geq 0}$ be an admissible sequence, $1<p \leq \infty, K \geq 0$, $c>0$. A function $a_{k m}: \mathbb{R}^{n} \rightarrow \mathbb{C}$ such that $D^{\alpha} a_{k m}$ exists for all $|\alpha| \leq \bar{K}$ is called an $(\sigma, p)_{K}-N$-atom if

$$
\begin{gathered}
\operatorname{supp} a_{k m} \subset Q_{k m} \quad \text { for some } k \in \mathbb{N}_{0}, m \in \mathbb{Z}^{n}, \\
\left|D^{\alpha} a_{k m}(x)\right| \leq \sigma_{k}^{-1} N_{k}^{n / p+|\alpha|}, \quad|\alpha| \leq K .
\end{gathered}
$$

The atomic decomposition theorem states that $g \in S^{\prime}\left(\mathbb{R}^{n}\right)$ belongs to $F_{p q}^{\sigma, N}\left(\mathbb{R}^{n}\right)$ (respectively, $B_{p q}^{\sigma, N}\left(\mathbb{R}^{n}\right)$ ) if and only if it can be represented as

$$
g=\sum_{k=0}^{\infty} \sum_{m \in \mathbb{Z}^{n}} \lambda_{k m} a_{k m}
$$

which is convergent in $S^{\prime}\left(\mathbb{R}^{n}\right)$, where $a_{k m}$ are $(\sigma, p)_{K}-N$-atoms, and $\lambda \in f_{k m}^{N}$ (respectively, $\lambda_{k m} \in b_{k m}$ ). For a more general statement we refer to [9].

Next we need the definitions of quarks and quarkonial decompositions. We need to adapt the construction introduced in [31, $\S 2$ and $\S 9]$ for ordinary Besov and Triebel-Lizorkin spaces to spaces of generalized smoothness. We will show later that quarkonial decomposition of a function is more convenient when we want to restrict this function to an $h$-set. 
Definition 7. Let $k \in \mathbb{N}_{0}$, and $\left\{x^{k m}: m \in \mathbb{Z}^{n}\right\} \subset \mathbb{R}^{n}$ be an approximate lattice such that there exist $c_{1}, c_{2} \geq 0$ for which

$$
\left|x^{k m_{1}}-x^{k m_{2}}\right| \geq c_{1} / N_{k}, \quad k \in \mathbb{N}_{0}, m_{1} \neq m_{2},
$$

and

$$
\mathbb{R}^{n}=\bigcup_{m \in \mathbb{Z}^{n}} B\left(x^{k m}, c_{2} / N_{k}\right), \quad k \in \mathbb{N}_{0}, c_{2}=c N_{r},
$$

for some fixed $r$, and a constant $c$. Here $B(x, d)$ is the ball centered at $x$ with radius $d$.

Let $\left\{\theta^{k m}: m \in \mathbb{Z}^{n}\right\}$ be the subordinate resolution of unity, i.e. $\theta^{k m}$ are non-negative $C^{\infty}$-functions in $\mathbb{R}^{n}$, supp $\theta^{k m} \subset B\left(x^{k m}, c_{2} / N_{k}\right), k \in \mathbb{N}_{0}$, $m \in \mathbb{Z}^{n}$, and

$$
\sum_{m \in \mathbb{Z}^{n}} \theta^{k m}(x)=1, \quad x \in \mathbb{R}^{n}, k \in \mathbb{N}_{0} .
$$

Since $\left\{\theta^{k m}\right\}$ is a resolution of unity, we have

$$
\left|N_{k}^{|\beta|}\left(x-x^{k m}\right)^{\beta} \theta^{k m}(x)\right| \leq c^{|\beta|} N_{r}^{|\beta|},
$$

where $\beta \in \mathbb{N}_{0}^{n}$, and $x^{\beta}=x_{1}^{\beta_{1}} \cdots x_{n}^{\beta_{n}}$. In addition we assume that

$$
\left|D^{\alpha} \theta^{k m}\right| \leq c_{\alpha} N_{k}^{|\alpha|}, \quad|\alpha| \leq K \text { for some large } K .
$$

Definition 8. Let $\beta \in \mathbb{N}_{0}^{n}, 1<p \leq \infty$. Then we will call the function

$$
(\beta q u)_{k m}^{\sigma, N}(x)=\sigma_{k}^{-1} N_{k}^{n / p+|\beta|}\left(x-x^{k m}\right)^{\beta} \theta^{k m}(x), \quad x \in \mathbb{R}^{n},
$$

a generalized $(N, \sigma, p, \beta)$-quark.

To shorten the notation we will call the quarks from Definition 8 simply $(N, \sigma)$-quarks.

Definition 9. Let $1<p \leq \infty, 1<q \leq \infty,\left(\sigma_{j}\right)_{j \geq 0}$ be an admissible sequence, and $\left(N_{j}\right)_{j \geq 0}$ be a strongly increasing sequence. Let $(\beta q u)_{k m}^{\sigma, N}$ be $(N, \sigma)$-quarks according to Definition 8 . We put

$$
\lambda=\left\{\lambda^{\beta}: \beta \in \mathbb{N}_{0}^{n}\right\}, \quad \lambda^{\beta}=\left\{\lambda_{k m}^{\beta} \in \mathbb{C}: k \in \mathbb{N}_{0}, m \in \mathbb{Z}^{n}\right\} .
$$

Let $\varrho>r$, where $r$ is from Definition $7, \lambda^{\beta} \in b_{p q}$, and

$$
\left\|\lambda\left|b_{p q}\left\|_{\varrho}=\sup _{\beta \in \mathbb{N}_{0}^{n}} N_{\varrho}^{|\beta|}\right\| \lambda^{\beta}\right| b_{p q}\right\|<\infty .
$$

Then $\mathbf{B}_{p q}^{\sigma, N}\left(\mathbb{R}^{n}\right)$ is the collection of all $g \in S^{\prime}\left(\mathbb{R}^{n}\right)$ which can be represented as

$$
g(x)=\sum_{\beta \in \mathbb{N}_{0}^{n}} \sum_{k=0}^{\infty} \sum_{m \in \mathbb{Z}^{n}} \lambda_{k m}^{\beta}(\beta q u)_{k m}^{\sigma, N}(x),
$$

where $\lambda$ satisfies (13). Furthermore,

$$
\left\|g\left|\mathbf{B}_{p q}^{\sigma, N}\left(\mathbb{R}^{n}\right)\left\|_{\theta, \varrho}=\inf \right\| \lambda\right| b_{p q}\right\|_{\varrho}
$$


where the infimum is taken over all admissible representations. Similarly,

$$
\begin{aligned}
\left\|g \mid \mathbf{F}_{p q}^{\sigma, N}\left(\mathbb{R}^{n}\right)\right\|_{\theta, \varrho} & =\inf \left\|\lambda \mid f_{p q}^{N}\right\|_{\varrho}, \quad \text { where } \\
\left\|\lambda \mid f_{p q}^{N}\right\|_{\varrho} & =\sup _{\beta \in \mathbb{N}_{0}^{n}} N_{\varrho}^{|\beta|}\left\|\lambda^{\beta} \mid f_{p q}^{N}\right\|<\infty .
\end{aligned}
$$

The spaces defined in Definitions 9 and 3 are equivalent as normed spaces:

Theorem 10. Let $1<p<\infty, 1<q<\infty,\left(\sigma_{j}\right)_{j \geq 0}$ be an increasing admissible sequence, and $\left(N_{j}\right)_{j \geq 0}$ be a strongly increasing sequence. Let $g \in$ $S^{\prime}\left(\mathbb{R}^{n}\right)$. Then

$$
\left\|g\left|F_{p q}^{\sigma, N}\left(\mathbb{R}^{n}\right)\|\sim\| g\right| \mathbf{F}_{p q}^{\sigma, N}\left(\mathbb{R}^{n}\right)\right\|_{\theta, \varrho}
$$

$$
\left\|g\left|B_{p q}^{\sigma, N}\left(\mathbb{R}^{n}\right)\|\sim\| g\right| \mathbf{B}_{p q}^{\sigma, N}\left(\mathbb{R}^{n}\right)\right\|_{\theta, \varrho} .
$$

For the proof see Appendix I.

2.2. Quarkonial representations on an h-set. Now we switch to the fractal case. We need to modify our generalized lattice in order to have convenient representations of the trace of a function which is initially defined on $\mathbb{R}^{n}$.

Definition 11. Let $\Gamma$ be a compact set in $\mathbb{R}^{n}$ and let

$$
\Gamma_{\varepsilon}=\left\{x \in \mathbb{R}^{n}: \operatorname{dist}(x, \Gamma)<\varepsilon\right\}, \quad \varepsilon>0,
$$

be the $\varepsilon$-neighborhood of $\Gamma$. Let $k \in \mathbb{N}_{0}$ and

$$
\left\{\gamma^{k m}: m=1, \ldots, M_{k}\right\} \subset \Gamma, \quad\left\{\theta^{k m}: m=1, \ldots, M_{k}\right\}
$$

be an approximate lattice and the subordinate resolution of unity with the following properties: there exist $c_{0}, c_{1}, c_{2}$ with

$$
\left|\gamma^{k m_{1}}-\gamma^{k m_{2}}\right| \geq c_{1} / N_{k}, \quad k \in \mathbb{N}_{0}, m_{1} \neq m_{2},
$$

and

$$
\Gamma_{\varepsilon_{k}} \subset \bigcup_{m=1}^{M_{k}} B\left(\gamma^{k m}, c_{2} / N_{k}\right), \quad k \in \mathbb{N}_{0},
$$

where $\varepsilon_{k}=c_{0} N_{k}^{-1}$. Further, let $\left\{\theta^{k m}\right\}$ be non-negative $C^{\infty}$-functions in $\mathbb{R}^{n}$,

$$
\operatorname{supp} \theta^{k m} \subset B\left(\gamma^{k m}, c_{2} / N_{k}\right), \quad k \in \mathbb{N}_{0}, m=1, \ldots, M_{k},
$$

and

$$
\left|D^{\alpha} \theta^{k m}(x)\right| \leq c_{\alpha} N_{k}^{|\alpha|}, \quad k \in \mathbb{N}_{0}, m=1, \ldots, M_{k},
$$

for all $\alpha \in \mathbb{N}_{0}^{n}$ and suitable constants $c_{\alpha}$, and

$$
\sum_{m=1}^{M_{k}} \theta^{k m}(x)=1, \quad x \in \Gamma_{\varepsilon_{k}}, k \in \mathbb{N}_{0} .
$$


We always assume that the approximate lattice $\left\{\gamma^{k m}\right\}$ and the decomposition of unity $\left\{\theta^{k m}\right\}$ can be extended to $\mathbb{R}^{n}$ so that one gets the approximate lattice $\left\{x^{k m}\right\}$ and the decomposition of unity $\left\{\theta^{k m}\right\}$ from Definition 7 (see also $[31, \S 9.24])$.

Later on we will obtain the quarkonial representation of a function on $\Gamma$ from its quarkonial representation on $\mathbb{R}^{n}$ by index shifting in $\left(\sigma_{k}\right)_{k \geq 0}$ (see $(12))$. In other words, we want to know the sequence $\left(\sigma_{k}^{*}\right)_{k \geq 0}$ to which $\left(\sigma_{k}\right)_{k \geq 0}$ will reduce when we switch from $\mathbb{R}^{n}$ to $\Gamma$.

Let $\mu$ be an $h$-measure, i.e. $\mu\left(B_{k m}\right) \sim h\left(1 / N_{k}\right)$. Then from $\sum_{m=1}^{M_{k}} \mu\left(B_{k m}\right)$ $\sim 1$ we get $M_{k} \sim 1 / h\left(1 / N_{k}\right), k \geq 1$.

Next we define the generalized quarks on $\Gamma$.

Definition 12. Let $\Gamma$ be an $h$-set, $\left\{\theta^{k m}\right\}$ be a resolution of unity introduced in Definition 11, $1<p \leq \infty,\left(\sigma_{j}^{*}\right)_{j \geq 0}$ be an admissible sequence, and $\left(N_{j}\right)_{j \geq 0}$ be a strongly increasing sequence. Then the function

$$
(\beta q u)_{k m}^{\sigma^{*}, N}(\gamma)=\left(\sigma_{k}^{*}\right)^{-1} h\left(1 / N_{k}\right)^{-1 / p} N_{k}^{|\beta|}\left(\gamma-\gamma^{k m}\right)^{\beta} \theta^{k, l}(\gamma), \quad \beta \in \mathbb{N}_{0}^{n},
$$

where $k \in \mathbb{N}_{0}$ and $m=1, \ldots, M_{k}$, is called a generalized $\left(N, \sigma^{*}, p, \beta\right)$-quark on $\Gamma$.

To shorten the notation, we will call a generalized $\left(N, \sigma^{*}, p, \beta\right)$-quark an $\left(N, \sigma^{*}\right)$-quark on $\Gamma$.

Analogously to $b_{p q}$ from Definition 5 , define for $1<p<\infty, 1<q \leq \infty$ the sequence space

$$
b_{p q}^{\Gamma}=\left\{\lambda:\left\|\lambda \mid b_{p q}^{\Gamma}\right\|=\left(\sum_{k=0}^{\infty}\left(\sum_{m=1}^{M_{k}}\left|\lambda_{k m}\right|^{p}\right)^{q / p}\right)^{1 / q}<\infty\right\} .
$$

Definition 13. Let $\Gamma$ be an $h$-set, $\left(\sigma_{j}^{*}\right)_{j \geq 0}$ be an admissible sequence, $\left(N_{j}\right)_{j \geq 0}$ be a strongly increasing sequence, $0<q \leq \infty, 1<p<\infty$, and let $(\beta q u)_{k m}^{\sigma^{*}, N}$ be $\left(N, \sigma^{*}\right)$-quarks on $\Gamma$, according to Definition 12. We put

$$
\lambda=\left\{\lambda^{\beta}: \beta \in \mathbb{N}_{0}^{n}\right\}, \quad \lambda^{\beta}=\left\{\lambda_{k m}^{\beta} \in \mathbb{C}: \nu \in \mathbb{N}_{0}, m=1, \ldots, M_{k}\right\} .
$$

Let $\varrho>r$, where $r$ is such that $c_{2}=c N_{r}, 0<c<1$ (see Definition 11), $\lambda^{\beta} \in b_{p q}^{\Gamma}$, and

$$
\left\|\lambda\left|b_{p q}^{\Gamma}\left\|_{\varrho}=\sup _{\beta \in \mathbb{N}_{0}^{n}} N_{\varrho}^{|\beta|}\right\| \lambda^{\beta}\right| b_{p q}^{\Gamma}\right\|<\infty .
$$

Then $B_{p q}^{\sigma^{*}, N}(\Gamma)_{\varrho}$ is the collection of all functions $g \in L_{1}(\Gamma)$ which can be represented as

$$
g(x)=\sum_{\beta \in \mathbb{N}_{0}^{n}} \sum_{k=0}^{\infty} \sum_{m=1}^{M_{k}} \lambda_{k m}^{\beta}(\beta q u)_{k m}^{\sigma^{*}, N}(\gamma)
$$


with (23). Furthermore,

$$
\left\|g\left|B_{p q}^{\sigma^{*}, N}(\Gamma)_{\varrho}\left\|_{\theta, \varrho}=\inf \right\| \lambda\right| b_{p q}^{\Gamma}\right\|_{\varrho}
$$

where the infimum is taken over all admissible representations.

To prove the trace theorem we need first to show that under some condition on $\left(\sigma_{j}^{*}\right)_{j \geq 0}$ the space $B_{p q}^{\sigma^{*}, N}(\Gamma)_{\varrho}$ is embedded in $L_{1}(\Gamma)$. The following lemma is a generalization of Proposition 9.31 from [31].

Lemma 14. Let the space $B_{p q}^{\sigma^{*}, N}(\Gamma)_{\varrho}$ be as in Definition 12, and $\left(\left(\sigma_{j}^{*}\right)^{-1}\right)_{j \geq 0} \in l_{q^{\prime}}$. Then the series $(24)$ converges in $L_{1}(\Gamma)$, and

$$
B_{p q}^{\sigma^{*}, N}(\Gamma)_{\varrho} \subset L_{1}(\Gamma) \quad \text { continuously. }
$$

Proof. Let $1<p<\infty, 1<q \leq \infty, \varrho>r$. Then, since

we have

$$
\left|N_{k}^{|\beta|}\left(\gamma-\gamma^{k m}\right)^{\beta} \theta^{k m}(\gamma)\right| \leq N_{r}^{|\beta|},
$$

$$
\begin{aligned}
& \int_{\Gamma}|g(\gamma)| \mu(d \gamma) \\
& \leq \sum_{\beta, k, m}\left|\lambda_{k m}^{\beta}\right|\left(\sigma_{k}^{*}\right)^{-1} h\left(1 / N_{k}\right)^{-1 / p} N_{k}^{|\beta|} \int_{\Gamma}\left|\left(\gamma-\gamma^{k m}\right)^{\beta} \theta^{k m}(\gamma)\right| \mu(d \gamma) \\
& \leq \sum_{\beta, k, m}\left(\sigma_{k}^{*}\right)^{-1} h\left(1 / N_{k}\right)^{-1 / p} N_{r}^{|\beta|} \mu\left(B_{k m}\right)\left|\lambda_{k m}^{\beta}\right| \\
& \leq \sum_{\beta \in \mathbb{N}_{0}^{n}} N_{r}^{|\beta|}\left\|\lambda^{\beta} \mid b_{p q}^{\Gamma}\right\|\left(\sum_{k=0}^{\infty}\left(\left(\sigma_{k}^{*}\right)^{-1} h\left(1 / N_{k}\right)^{-1 / p}\right)^{q^{\prime}}\left(\sum_{m=1}^{M_{k}} \mu\left(B_{k m}\right)^{p^{\prime}}\right)^{q^{\prime} / p^{\prime}}\right)^{1 / q^{\prime}} \\
& \leq \sup _{\beta \in \mathbb{N}_{0}^{n}} N_{\varrho}^{|\beta|}\left\|\lambda^{\beta} \mid b_{p q}^{\Gamma}\right\|\left(\sum_{j \geq 0}\left(\sigma_{j}^{*}\right)^{-q^{\prime}}\right)^{1 / q^{\prime}},
\end{aligned}
$$

where we used the fact that $\left(\sum_{m=1}^{M_{k}} \mu\left(B_{k m}\right)^{p^{\prime}}\right)^{1 / p^{\prime}} \sim M_{k}^{-1 / p} \sim h^{1 / p}\left(1 / N_{k}\right)$.

We can now define the trace operator. Let $\varphi \in S\left(\mathbb{R}^{n}\right)$; then we denote the pointwise trace of $\varphi$ on $\Gamma$ by $\operatorname{tr}_{\Gamma} \varphi$. If there is a constant $C>0$ such that for some $\tau \geq 1$,

$$
\left\|\operatorname{tr}_{\Gamma} \varphi\left|L_{\tau}(\Gamma)\|\leq C\| \varphi\right| B_{p q}^{\sigma, N}\left(\mathbb{R}^{n}\right)\right\|
$$

for all $\varphi \in S\left(\mathbb{R}^{n}\right)$, then we call the continuous extension $\operatorname{tr}_{\Gamma}$ of this mapping to $B_{p q}^{\sigma, N}\left(\mathbb{R}^{n}\right)$ the trace operator.

Write

$$
\operatorname{tr}_{\Gamma} B_{p q}^{\sigma, N}\left(\mathbb{R}^{n}\right)=\left\{u \in L_{1}(\Gamma): \text { there exists } g \in B_{p q}^{\sigma, N}\left(\mathbb{R}^{n}\right), \operatorname{tr}_{\Gamma} g=u\right\}
$$
with the norm

$$
\left\|u\left|\operatorname{tr}_{\Gamma} B_{p q}^{\sigma, N}\left(\mathbb{R}^{n}\right)\|=\inf \| g\right| B_{p q}^{\sigma, N}\left(\mathbb{R}^{n}\right)\right\|,
$$

where the infimum is taken over all $g \in B_{p q}^{\sigma, N}\left(\mathbb{R}^{n}\right)$ with $\operatorname{tr}_{\Gamma} g=u$. 
We arrive at the main theorem of this section:

Theorem 15. Let $\Gamma$ be an h-set, $1<p<\infty, 0<q \leq \infty,\left(N_{j}\right)_{j \geq 0}$ be a strongly increasing sequence, and $\sigma=\left(\sigma_{j}\right)_{j \geq 0}$ be an admissible sequence such that

$$
\sum_{j \in \mathbb{N}_{0}}\left(\sigma_{j} N_{j}^{-n / p} h\left(1 / N_{j}\right)^{-1 / p}\right)^{-q^{\prime}}<\infty .
$$

Consider the sequence $\sigma_{j}^{*}=\sigma_{j} N_{j}^{-n / p} h\left(1 / N_{j}\right)^{-1 / p}, j \in \mathbb{N}_{0}$. Then the spaces $B_{p q}^{\sigma^{*}, N}(\Gamma)_{\varrho}$ are independent of all allowed resolutions of unity and all allowed numbers $\varrho$, and will be denoted by $B_{p q}^{\sigma^{*}, N}(\Gamma)$, and

$$
\operatorname{tr}_{\Gamma} B_{p q}^{\sigma, N}\left(\mathbb{R}^{n}\right)=B_{p q}^{\sigma^{*}, N}(\Gamma) .
$$

Proof. Consider $g \in B_{p q}^{\sigma, N}\left(\mathbb{R}^{n}\right)$. By Theorem 10, $g$ admits a generalized quarkonial representation. Assume that the resolution of unity (9) is adapted to $\Gamma$, as described in Definition 11. If we take a finite sum of this representation, which is a smooth function $\varphi$, its restriction to $\Gamma$ admits a quarkonial representation (24). Therefore, by Lemma 14, Theorem 10 and (26) for our $\varphi$ and $\tau=1$, by continuous extension, $\operatorname{tr}_{\Gamma} g$ exists and

$$
\left\|\operatorname{tr}_{\Gamma} g\left|B_{p q}^{\sigma^{*}, N}(\Gamma)_{\varrho}\|\leq C\| g\right| B_{p q}^{\sigma, N}\left(\mathbb{R}^{n}\right)\right\| .
$$

On the other hand, let $u \in B_{p q}^{\sigma^{*}, N}(\Gamma)_{\varrho}$. By shifting we interpret it as a function on $\mathbb{R}^{n}$. Then, denoting by $g=\operatorname{ext} u$ the extension from $\Gamma$ to $\mathbb{R}^{n}$, we obtain

$$
\left\|g\left|B_{p q}^{\sigma, N}\left(\mathbb{R}^{n}\right)\left\|_{\varrho} \leq\right\| u\right| B_{p q}^{\sigma^{*}, N}(\Gamma)_{\varrho}\right\| .
$$

By equivalence of the norms $\|\cdot\|_{\varrho}$ for all $\varrho>r$, we obtain the statement of the theorem.

\section{Representation via differences. Trace theorem II}

3.1. Representation via differences on $\mathbb{R}^{n}$. In this part we find a different representation of spaces of generalized smoothness, and of their trace spaces on $h$-sets. To do this we need a theorem similar to Theorem 2.4.1 from [29] for spaces of generalized smoothness. For this we specify the sequence $\sigma$ and assume some additional conditions on the Bernstein function $f$ (compare with the introduction). For an analogous result see also [13] and [25].

Theorem 16. Let $\varphi$ be a complex-valued $C^{\infty}$ function on $\mathbb{R}^{n} \backslash\{0\}$ such that $|\varphi(x)|>0$ for $c^{-1}<|x|<c, c>0$. Let $\sigma=\left(2^{j \alpha}\right)_{j \geq 0}$, and $N=\left(N_{j}\right)_{j \geq 0}$, $N_{j}=\sqrt{f^{-1}\left(2^{2 j}\right)}$. Let $a>n / \min (p, q), \alpha_{0} \kappa<\alpha<\alpha_{1} \kappa$, where $\kappa$ is from condition (3) for $\left(N_{j}\right)_{j \geq 0}$, and suppose that

$$
\int_{\mathbb{R}^{n}}\left|\left(\frac{\varphi(\cdot) M(\cdot)}{|\cdot|^{\alpha_{1}}}\right)^{\vee}(y)\right|(1+|y|)^{a} d y<\infty
$$


and

$$
\sup _{l} \sup _{j} 2^{-l \kappa \alpha_{0}} \int_{\mathbb{R}^{n}}\left(\varphi\left(\frac{N_{j+l}}{N_{j}} \cdot\right) H(\cdot)\right)^{\vee}(y)(1+|y|)^{a} d y<\infty,
$$

where $M, H \in S\left(\mathbb{R}^{n}\right)$ are such that

$$
\operatorname{supp} M \subset\left\{y \in \mathbb{R}^{n}:|y| \leq 2\right\}, \quad M(x)=1 \quad \text { if }|x| \leq 1,
$$

and

$\operatorname{supp} H \subset\left\{y \in \mathbb{R}^{n}: 2^{-\kappa-1} \leq|y| \leq 2^{\kappa+1}\right\}, \quad H(x)=1 \quad$ if $2^{-\kappa} \leq|y| \leq 2^{\kappa}$.

Put $\varphi_{j}(x)=\varphi\left(N_{j}^{-1} x\right), x \in \mathbb{R}^{n} \backslash\{0\}, j \in \mathbb{N}_{0}$. Then for $1<p, q<\infty$,

$$
\left\|\left(\sum_{j=0}^{\infty} 2^{j \alpha q}\left|\varphi_{j}(D) u(\cdot)\right|^{q}\right)^{1 / q} \mid L_{p}\left(\mathbb{R}^{n}\right)\right\|
$$

and

$$
\left\|u\left|L_{p}\|+\|\left(\int_{0}^{1} \frac{\left|\varphi\left(D / \sqrt{f^{-1}\left(1 / t^{2}\right)}\right) u(\cdot)\right|^{q} d t}{t^{1+\alpha q}}\right)^{1 / q}\right| L_{p}\left(\mathbb{R}^{n}\right)\right\|
$$

are equivalent (quasi-)norms in $F_{p q}^{\sigma, N}\left(\mathbb{R}^{n}\right)$.

For the proof see Appendix II.

For convenience we rewrite (34), making the necessary variable change. We obtain

$$
\begin{aligned}
& \left\|u \mid L_{p}\left(\mathbb{R}^{n}\right)\right\| \\
& \quad+\left\|\left(\int_{0}^{1} \frac{\left|f\left(1 /|s|^{2}\right)^{\alpha / 2} \varphi(s \cdot D) u(\cdot)\right|^{q}}{s} \frac{f^{\prime}\left(1 / s^{2}\right)}{s^{2} f\left(1 / s^{2}\right)} d s\right)^{1 / q} \mid L_{p}\left(\mathbb{R}^{n}\right)\right\| .
\end{aligned}
$$

Since for Bernstein functions the inequality

$$
\left|f^{(k)}(t)\right| \leq \frac{k ! f(t)}{t^{k}}
$$

holds (see [15]), and $f^{\prime} \geq 0$, we see that (34) is equivalent to the norm

$$
\left\|u\left|L_{p}\left(\mathbb{R}^{n}\right)\|+\|\left(\int_{0}^{1} \frac{\left|f\left(1 /|s|^{2}\right)^{\alpha / 2} \varphi(s \cdot D) u(\cdot)\right|^{q}}{s} d s\right)^{1 / q}\right| L_{p}\left(\mathbb{R}^{n}\right)\right\|
$$

By the same arguments as in Theorem 2.6.1 and Remark 2.6.1/2 of [29] we deduce that for $p=q$ the norm (36) is equivalent to

$$
\left\|u \mid L_{p}\left(\mathbb{R}^{n}\right)\right\|+\left(\int_{|s| \leq 1} f\left(1 /|s|^{2}\right)^{\alpha p / 2}\left\|\triangle_{s}^{k} u\right\|_{p}^{p} \frac{d s}{|s|^{n}}\right)^{1 / p}
$$

where $\triangle_{s}^{k}$ is the $k$ th order difference, and $k>\alpha$. 
For example, let $p=2$ and $0<\alpha<1$ (if there exists $\varepsilon>0$ such that $f(x)<x^{1-\varepsilon}$ as $x \rightarrow \infty$, we can allow $\alpha=1$ ); then we can take $k=1$. Under such restrictions the norm in $B_{22}^{\sigma, N}\left(\mathbb{R}^{n}\right)$, where $\sigma=\left(2^{\alpha j}\right)_{j \geq 0}$, and $N=\left(N_{j}\right)_{j \geq 0}, N_{j}=\sqrt{f^{-1}\left(2^{2 j}\right)}$, is equivalent to

$$
\left\|u \mid L_{2}\left(\mathbb{R}^{n}\right)\right\|+\left(\iint_{|x-y|<1} f\left(\frac{1}{|x-y|^{2}}\right)^{\alpha}|u(y)-u(x)|^{2} \frac{d x d y}{|x-y|^{n}}\right)^{1 / 2}
$$

We would like to remark that in [17] an expression equivalent to (38) was obtained by completely different methods.

3.2. Trace theorem II. Our next aim will be to find, using (38), the representation for the norm in the trace space on an $h$-set. For classical Besov spaces and a generalized version of $h$-sets such a result was presented in [19].

Let $\mu$ be an $h$-measure, $\alpha$ be as in Theorem 18 (see below),

$$
\begin{aligned}
& \|u\|^{\alpha, f}:=\left\|u \mid L_{p}(\Gamma)\right\| \\
& \quad+\left(\sum_{\nu=0}^{\infty} \frac{f\left(2^{2 \nu}\right)^{\alpha p / 2} 2^{-\nu n}}{h\left(2^{-\nu}\right)^{2}} \iint_{|x-y| \leq 2^{-\nu}}|u(x)-u(y)|^{p} \mu(d x) \mu(d y)\right)^{1 / p},
\end{aligned}
$$

and consider the space $\mathcal{B}_{p p}^{\alpha, f}(\Gamma)=\left\{u \in S^{\prime}\left(\mathbb{R}^{n}\right):\|u\|^{\alpha, f}<\infty\right\}$. We will show in the next section that this space is equivalent to the trace space $B_{p p}^{\sigma^{*}, N}(\Gamma)$, defined in (13).

We need some notations; see [19] and [20] for details.

Let $\left\{Q_{i}\right\}$ be a collection of closed cubes with disjoint interiors, with sides parallel to the axes, and such that for the complement $\Gamma^{\mathrm{c}}$ of $\Gamma$ we have $\Gamma^{\mathrm{c}}=\bigcup Q_{i}$. Denote by $x_{i}$ the center of $Q_{i}$, and by $l_{i}$ and $s_{i}$ its diameter and side length. Let $0<\varepsilon<1 / 4$, and put $Q_{i}^{*}=(1+\varepsilon) Q_{i}$. We associate with this decomposition a partition of unity $\left\{\varphi_{i}\right\}$ such that $\varphi_{i}(x)=0$ if $x \notin Q_{i}^{*}$, $\sum \varphi_{i}(x)=1$ for $x \in \Gamma^{\mathrm{c}}$, and

$$
\left|D^{j} \varphi_{i}(x)\right| \leq a_{i} l_{i}^{-|j|} .
$$

Let $c_{i}=\mu\left(B\left(x_{i}, 6 l_{i}\right)\right)^{-1}$. On $\mathcal{B}_{p p}^{\alpha, f}(\Gamma)$ define the extension operator

$$
E u(x)=\sum_{i \in I} \varphi_{i}(x) c_{i} \int_{\left|t-x_{i}\right| \leq 6 l_{i}} u(t) d \mu(t), \quad x \in \Gamma^{\mathrm{c}},
$$

where $I=\left\{i: s_{i} \leq 1\right\}$.

Next we give another definition of the trace operator.

DeFinition 17. Let $u$ be a locally integrable function defined on $\mathbb{R}^{n}$, and $m$ be the Lebesgue measure on $\mathbb{R}^{n}$. The strictly defined function corre- 
sponding to $u$ is

$$
\widetilde{u}(x)=\lim _{r \rightarrow 0} \frac{1}{m(B(x, r))} \int_{B(x, r)} u(y) d y, \quad x \in \mathbb{R}^{n},
$$

if this limit exists. Then define the restriction operator

$$
R u=\left.\widetilde{u}\right|_{\Gamma}
$$

Now we show that restriction and extension between $\mathcal{B}_{p p}^{\alpha, f}(\Gamma)$ and $B_{p p}^{\sigma, N}\left(\mathbb{R}^{n}\right)$ are continuous, and correspond to each other. The continuity of the extension operator $E$ follows by the same arguments as in [19] and [20], but to prove the continuity of the restriction $R$ we need condition (4) on $f$.

THEOREM 18. Let $p>1$, let $f$ be a Bernstein function such that $f(t) t^{-1 / \kappa}$ increases for some $\kappa \in[1, \infty)$ as $t \rightarrow \infty$, and

$$
\frac{1}{c^{\delta}} \leq \frac{f(c t)}{f(t)} \leq c \quad \text { for all } t>0, c \geq 1 \text { and some } 0<\delta<1 \text {. }
$$

Assume in addition that $\alpha$ is such that

$$
x^{(n-d) / p}<f^{\alpha / 2}\left(x^{2}\right)<x^{(n-s) / p+1}
$$

for large $x$ and suitable $0<d \leq s \leq n$, and for $\delta$ we have $0<\delta \alpha<1$. Let $\Gamma$ be an $h$-set, where $h$ satisfies, for all $0<t, \lambda<1$,

$$
c_{1} \lambda^{s} \leq \frac{h(\lambda t)}{h(t)} \leq c_{2} \lambda^{d} \quad \text { for some } c_{1}, c_{2}>0
$$

Then the restriction operator $R$, defined in (42), is continuous from $B_{p p}^{\sigma, N}\left(\mathbb{R}^{n}\right), 1<p<\infty, N=\left(N_{j}\right)_{j \geq 0}, N_{j}=\sqrt{f^{-1}\left(2^{2 j}\right)}, \sigma=\left(2^{\alpha j}\right)_{j \geq 0}$, to $\mathcal{B}_{p p}^{\alpha, f}(\Gamma)$, and there exists a continuous extension $E$ from $\mathcal{B}_{p p}^{\alpha, f}(\Gamma)$ to $B_{p p}^{\sigma, N}\left(\mathbb{R}^{n}\right)$, which can be defined by $(40)$.

The proof is given in Appendix III.

REMARK 19. The representation (39) is the discrete version of

$$
\begin{aligned}
& \left\|u \mid L_{p}(\Gamma)\right\| \\
& \quad+\left(\iint_{|x-y|<1} \frac{f\left(|x-y|^{-2}\right)^{\alpha p / 2}}{|x-y|^{-n} h(|x-y|)^{2}}|u(x)-u(y)|^{p} \mu(d x) \mu(d y)\right)^{1 / p} ;
\end{aligned}
$$

see Proposition 2 in [19].

REMARK 20. For the classical case, i.e. when $f^{\alpha / 2}\left(x^{2}\right)=x^{\alpha}$, condition (43) coincides with the condition given in [19]: $(n-d) / p<\alpha<(n-s) / p+1$, where $s$ and $d$ have the same meaning as in Theorem 18 . 
3.3. Equivalence theorem. In this subsection we show the equivalence of the trace spaces $B_{p p}^{\sigma^{*}, N}(\Gamma)$ and $\mathcal{B}_{p p}^{\alpha, f}(\Gamma)$.

Let $\sigma=\sigma^{\alpha}=\left(2^{j \alpha}\right)_{j \geq 0}, \alpha>0$. To show that $u=\widetilde{u} \mu$-a.e. for $u \in$ $B_{p q}^{\sigma, N}\left(\mathbb{R}^{n}\right)$ and $\widetilde{u}$ as defined in (41), we need the notions of $(r, p)$ - $\psi$-capacity of a set (see [16] and [18]), where $\psi$ is a continuous negative-definite function. Let $G$ be an open set in $L_{p}\left(\mathbb{R}^{n}\right)$, and $r>0$. The set function

$$
\operatorname{Cap}_{r, p}^{\psi}(G)=\inf \left\{\left\|u \mid H_{p}^{\psi, r}\left(\mathbb{R}^{n}\right)\right\|: u \geq 1 \text { a.e. on } G\right\}
$$

is called the $(r, p)$ - $\psi$-capacity of an open set $G$.

For an arbitrary set $A \subset \mathbb{R}^{n}$ the capacity is defined as

$$
\operatorname{Cap}_{r, p}^{\psi}(A):=\inf \left\{\operatorname{Cap}_{r, p}^{\psi}(G): A \subset G, G \subset \mathbb{R}^{n} \text { open }\right\} .
$$

Proposition 21. Let $\left(\sigma_{j}\right)_{j \geq 0}$ satisfy (29). Then $g=\widetilde{g} \mu$-a.e. for $g \in$ $B_{p q}^{\sigma, N}\left(\mathbb{R}^{n}\right)$.

Proof. Since $\left(\sigma_{j}\right)_{j \geq 0}$ satisfies (29), the operator $\operatorname{tr}_{\Gamma}$ is continuous from $B_{p q}^{\sigma, N}\left(\mathbb{R}^{n}\right)$ to $L_{p}(\Gamma), p \geq 1$. By the same arguments as in $\S 2.3 .2$ of [28], and $\S 2.3 .3$ of [27], for all $0<q \leq \infty$ and $\alpha>0$ we get

$$
B_{p q}^{\sigma^{\alpha}, N}\left(\mathbb{R}^{n}\right) \subset B_{p 2}^{\sigma^{\alpha-\varepsilon}, N}\left(\mathbb{R}^{n}\right) \subset F_{p 2}^{\sigma^{\alpha-\varepsilon}, N}\left(\mathbb{R}^{n}\right)=H_{p}^{f\left(|\cdot|^{2}\right), \alpha-\varepsilon}\left(\mathbb{R}^{n}\right),
$$

for $p \geq 2,0<q \leq \infty$, and

$$
B_{p q}^{\sigma^{\alpha}, N}\left(\mathbb{R}^{n}\right) \subset B_{p p}^{\sigma^{\alpha-\varepsilon}, N}\left(\mathbb{R}^{n}\right) \subset F_{p 2}^{\sigma^{\alpha-\varepsilon}, N}\left(\mathbb{R}^{n}\right)=H_{p}^{f\left(|\cdot|^{2}\right), \alpha-\varepsilon}\left(\mathbb{R}^{n}\right)
$$

for $p<2$. Since by Theorem 3.1.47 of [16] any function from $H_{p}^{\psi, r-\varepsilon}\left(\mathbb{R}^{n}\right)$ admits an $(r-\varepsilon, p)$ - $\psi$-quasi-continuous modification, any function $u \in B_{p q}^{\sigma^{\alpha}, N}\left(\mathbb{R}^{n}\right)$ can be strictly defined up to a set of $(\alpha-\varepsilon, p)-f\left(|\cdot|^{2}\right)$-capacity zero for all $\varepsilon>0$. Let $\Gamma_{0} \subset \Gamma, \varphi \in S\left(\mathbb{R}^{n}\right), \varphi \geq 1$ on $\Gamma_{0}$ and $\left\|\varphi \mid B_{p q}^{\sigma, N}\left(\mathbb{R}^{n}\right)\right\|<\delta$ for some $\delta>0$. Then

$$
\mu\left(\Gamma_{0}\right) \leq\left(\int_{\Gamma}|\varphi(\gamma)|^{p} d \mu\right)^{1 / p} \leq c\left\|\varphi \mid H_{p}^{f\left(|\cdot|^{2}\right), \alpha-\varepsilon}\left(\mathbb{R}^{n}\right)\right\|<\delta .
$$

Therefore a set of $(\alpha-\varepsilon, p)-f\left(|\cdot|^{2}\right)$-capacity zero also has $\mu$-measure zero. Thus $g=\widetilde{g} \mu$-a.e.

Remark 22. From the proof of Proposition 21 we get: if a set has $(\alpha, 2)$ $\psi$-capacity zero, then it has $\mu$-measure zero.

The following statements are modifications of Lemma 3.4.14 and Theorem 3.4.15 from [3].

Lemma 23. Suppose that $\left(\sigma_{j}\right)_{j \geq 0}$ satisfies (29). Consider a family $\left\{a_{j m}\right\}$ of $\left(\sigma_{j} h\left(1 / N_{j}\right)^{-1 / p} N_{j}^{-n / p}, p\right)_{K^{-a t o m s}}$ located at $c Q_{j m}$ from Definition 6 , and a sequence $\lambda=\left(\lambda_{j m}\right)_{j \geq 0, m \in \mathbb{Z}^{n}} \in b_{p q}$. For $k, M \in \mathbb{N}$ with $k>M$ define the 
function

$$
\left(g_{M}\right)_{k}(\gamma)=\left|\frac{1}{m\left(B\left(\gamma, N_{k}^{-1}\right)\right)} \int_{B\left(\gamma, N_{k}^{-1}\right)} \sum_{j=M+1}^{\infty} \sum_{m \in \mathbb{Z}^{n}} \lambda_{j m} a_{j m}(x) d x\right|^{p} .
$$

Then there exist $c_{1}$ and $\varepsilon>0$ such that

$$
\int_{\Gamma}\left(g_{M}\right)_{k}(\gamma) d \gamma \leq c_{1} 2^{-M \varepsilon} .
$$

From Lemma 23 we get

Theorem 24. Let $\left(\sigma_{j}\right)_{j \geq 0}$ satisfy (29). Then

$$
\operatorname{tr}_{\Gamma} g=R g \quad \text { for every } g \in B_{p q}^{\sigma, N}\left(\mathbb{R}^{n}\right) .
$$

The proofs are completely analogous to those given in [3], we only need to use (44).

Now we can state the equivalence theorem.

Theorem 25. Let $1<p<\infty, \Gamma$ be an h-set, $h$ satisfy (44), and $f$ be a Bernstein function satisfying $(4),(2)$, and (43). Then the spaces $\mathcal{B}_{p p}^{\alpha, f}(\Gamma)$ and $B_{p p}^{\sigma^{*}, N}(\Gamma)$, with $N=\left(N_{j}\right)_{j \geq 0}, N_{j}=\sqrt{f^{-1}\left(2^{2 j}\right)}$, and $\sigma^{*}=\left(\sigma_{j}^{*}\right)_{j \geq 0}$, $\sigma_{j}^{*}=\sigma_{j} h\left(c / N_{j}\right)^{-1 / p} N_{j}^{-n / p}$, are equivalent as normed spaces.

Proof. This follows from Theorems 15, 18 and 24.

\section{Application: Domain of the time-changed Dirichlet form. In} this part we show that the space $\mathcal{B}_{p p}^{\alpha, f}(\Gamma)$ is the domain of some time-changed Dirichlet form, which corresponds to a Markov process on an $h$-set.

We start with a general definition of a Dirichlet form (see [11]).

Let $(X, \mathcal{B}, m)$ be a $\sigma$-finite measurable space. A Dirichlet form $(\mathcal{E}, D(\mathcal{E}))$, where $D(\mathcal{E})$ is the domain of $\mathcal{E}$, is a symmetric form on $L_{2}(X, m)$ which is closed and Markovian. The latter means that

$$
u \in D(\mathcal{E}), v=(0 \vee u) \wedge 1 \quad \text { imply } \quad v \in D(\mathcal{E}) \text {, and } \mathcal{E}(v, v) \leq \mathcal{E}(u, u) .
$$

There is a one-to-one correspondence between symmetric Dirichlet forms and symmetric Markov processes, i.e. with a symmetric Dirichlet form $\mathcal{E}(\cdot, \cdot)$ one can associate a self-adjoint operator $-A$ such that

$$
\mathcal{E}(u, v)=(\sqrt{-A} u, \sqrt{-A} v)_{2},
$$

where $(\cdot, \cdot)_{2}$ is the scalar product in $L_{2}\left(\mathbb{R}^{n}\right)$. Then $-A$ is the generator of an $L_{2}$-sub-Markovian semigroup $\left(T_{t}\right)_{t \geq 0}$, which is associated with a Markov process $\left(X_{t}\right)_{t \geq 0}$ as follows:

$$
T_{t} u(x)=E_{x}\left(u\left(X_{t}\right)\right)
$$

Sometimes it is more convenient to study a Markov process using tools from functional analysis, i.e. Dirichlet forms. 
Let $\psi$ be a continuous negative-definite function; then it is an exponent of a Lévy process $\left(X_{t}\right)_{t \geq 0}$, i.e. if $X_{0}=0$, then

$$
\mathbb{E}_{0} e^{i \xi X_{t}}=\int_{\mathbb{R}^{n}} e^{i \xi x} p_{t}(x) d x=e^{-t \psi(\xi)} .
$$

On the other hand, let $A$ be an operator with symbol $\psi$, i.e. for $u \in S\left(\mathbb{R}^{n}\right)$ we have $\widehat{A u}(\xi)=\psi(\xi) \widehat{u}(\xi)$. Then

$$
\mathcal{E}(u, v)=\int_{\mathbb{R}^{n}} \psi(\xi) \widehat{u}(\xi) \widehat{v}(\xi) d \xi
$$

is the Dirichlet form associated with $\psi$. Its domain can be defined in terms of $\psi$-Bessel potential spaces.

Let $p>1, s>0$; the $\psi$-Bessel potential space $H_{p}^{\psi, s}\left(\mathbb{R}^{n}\right)$ is defined as (see [16])

$$
H_{p}^{\psi, s}\left(\mathbb{R}^{n}\right)=\left\{u \in S^{\prime}\left(\mathbb{R}^{n}\right):\left\|\left((1+\psi(\xi))^{s / 2} \widehat{u}(\xi)\right)^{\vee}(\cdot) \mid L_{p}\left(\mathbb{R}^{n}\right)\right\|<\infty\right\} .
$$

Then $D(\mathcal{E})=H_{2}^{\psi, 1}\left(\mathbb{R}^{n}\right)=: H^{\psi, 1}\left(\mathbb{R}^{n}\right)$.

Let $\Gamma$ be an $h$-set, and $\mu$ be some fixed $h$-measure on $\Gamma$. We want to describe the Markov process on $\Gamma$ which is obtained by restriction of $\left(X_{t}\right)_{t \geq 0}$ to $\Gamma$, i.e. we consider $\left(X_{t}\right)_{t \geq 0}$ at the random times when $\left(X_{t}\right)_{t \geq 0}$ hits $\Gamma$. The resulting process $\left(\check{X}_{t}\right)_{t \geq 0}$ is called the time-changed random process. We will describe the Dirichlet form which is associated with this process, and for this we apply the tools from function space theory, developed in the previous sections. For $d$-sets and stable-like jump processes such problems were studied in [26], [21], [22], [12].

First we need to find a condition under which it is possible to construct such a time-changed process.

Let $\sigma$ and $N$ be such that $B_{22}^{\sigma, N}\left(\mathbb{R}^{n}\right)=H^{\psi, 1}\left(\mathbb{R}^{n}\right)$. For example, if $\psi(\xi)=$ $f\left(|\xi|^{2}\right)$, we take $\sigma_{j}=2^{j}$ and $N_{j}=\sqrt{f^{-1}\left(2^{2 j}\right)}, j \geq 1$.

We claim: if $H^{\psi, 1}\left(\mathbb{R}^{n}\right)$ admits the trace space on $\Gamma$, then $\operatorname{Cap}_{r, p}^{\psi}(\Gamma)>0$.

Indeed, let $K \subset \mathbb{R}^{n}$ be a compact set such that $\Gamma \subset K$. Then there exists a function $u \in H^{\psi, 1}\left(\mathbb{R}^{n}\right)$ with $u \geq 1$ on $K$. Suppose that there exists the trace space of $H^{\psi, 1}\left(\mathbb{R}^{n}\right)$ on $\Gamma$. $\operatorname{Then}^{\operatorname{tr}_{\Gamma}} u \geq 1$ on $\Gamma$.

Let

$$
\mathcal{L}_{\Gamma}=\left\{u \in H^{\psi, 1}\left(\mathbb{R}^{n}\right): u \geq 1 \text { on } \Gamma\right\} .
$$

By Lemma 3.1.1 from [10] (or by Theorem 3.1.31 from [16]) there exists a unique element $e_{\Gamma} \in \mathcal{L}_{\Gamma}$ such that $0 \leq e_{\Gamma} \leq 1 \mu$-a.e., $e_{\Gamma}=1 \mu$-a.e. on $\Gamma$, and

$$
\operatorname{Cap}_{1,2}^{\psi}(\Gamma)=\left\|e_{\Gamma} \mid H^{\psi, 1}\left(\mathbb{R}^{n}\right)\right\|,
$$

which leads to $\operatorname{Cap}_{1,2}^{\psi}(\Gamma)>0$; otherwise $e_{\Gamma}=0$ a.e. 
By Remark $22, \mu$ charges no set of $(1,2)$ - $\psi$-capacity zero. Since $\operatorname{Cap}_{1,2}^{\psi}(\Gamma)$ $>0$, there exists (see $[11, \S 5.1]$ ) a positive continuous additive functional (PCAF) $\left(L_{t}\right)_{t \geq 0}$ which is in the Revuz correspondence with $\mu$.

Take $t(\tau):=\inf \left\{t: L_{t}>\tau\right\}$, i.e. the right inverse of $L_{t}$. Then $(t(\tau))_{\tau \geq 0}$ is an increasing process, and we put

$$
\left(\check{X}_{\tau}\right)_{\tau \geq 0}=\left(X_{t(\tau)}\right)_{\tau \geq 0} .
$$

Another way of constructing the time-changed process on a compact $d$-set $\Gamma$, using approximation by time-changed Markov processes on $\varepsilon$-parallel sets $\Gamma$, is described in [12].

Let $\check{\Gamma}$ be the support of $\left(L_{t}\right)_{t \geq 0}$, and denote by $\sigma_{\check{\Gamma}}$ the first moment when $\left(X_{t}\right)_{t \geq 0}$ hits $\check{\Gamma}$. Note that $\sigma_{\check{\Gamma}}<\infty$ a.s., since $\operatorname{Cap}_{1,2}^{\psi}(\check{\Gamma})>0$.

Define $\bar{H}_{\check{\Gamma}} u(x)=E_{x}\left(u\left(X_{\sigma_{\check{\Gamma}}}\right)\right)$.

REMARK 26. The time-changed Dirichlet form $(\check{\mathcal{E}}, \check{\mathcal{F}})$ associated with $\left(\check{X}_{t}\right)_{t \geq 0}$ can be obtained as follows:

$$
\begin{aligned}
& \check{\mathcal{F}}=\left\{\varphi \in L_{2}(\Gamma, \mu): \varphi=\operatorname{tr}_{\Gamma} u \mu \text {-a.e. on } \Gamma \text { for some } u \in H^{\psi, 1}\left(\mathbb{R}^{n}\right)\right\}, \\
& \check{\mathcal{E}}(\varphi, \varphi)=\mathcal{E}\left(H_{\check{\Gamma}} u, H_{\breve{\Gamma}} u\right), \quad \varphi \in \check{\mathcal{F}}, \varphi=\operatorname{tr}_{\Gamma} u \mu \text {-a.e. on } \Gamma, u \in H^{\psi, 1}\left(\mathbb{R}^{n}\right) .
\end{aligned}
$$

(See [11] for the general theory.)

By the Dirichlet principle (see [10]),

$$
\check{\mathcal{E}}(\varphi, \varphi)=\inf \left\{\mathcal{E}(u, u): u=\varphi \mu \text {-a.e. on } \Gamma, u \in H^{\psi, 1}\left(\mathbb{R}^{n}\right)\right\},
$$

we obtain $D(\check{\mathcal{E}})=\operatorname{tr}_{\Gamma} H^{\psi, 1}\left(\mathbb{R}^{n}\right)$.

For our case it is possible to define the domain of this time-changed Dirichlet form in terms of function spaces on $\Gamma$. Let $\psi(\xi)=f\left(|\xi|^{2}\right)$, and

$$
\mathcal{E}(u, v)=\int_{\mathbb{R}^{n}} f\left(|\xi|^{2}\right) \widehat{u}(\xi) \widehat{v}(\xi) d \xi .
$$

Due to the equivalence theorem (Theorem 25), we get not only the inclusion $\mathcal{B}_{22}^{1, f}(\Gamma) \subset \check{\mathcal{F}}$, but even $\mathcal{B}_{22}^{1, f}(\Gamma)=\check{\mathcal{F}}$. Thus, we arrive at the theorem which connects the theory of function spaces and the theory of Dirichlet forms:

Theorem 27. Let $\left(\mathcal{E}, H^{f\left(|\cdot|^{2}\right), 1}\left(\mathbb{R}^{n}\right)\right)$ be a Dirichlet form defined by $(47)$, $f$ be a Bernstein function such that $f(t) t^{-1 / \kappa}$ increases for some $\kappa \in[1, \infty)$ as $t \rightarrow \infty$, and

$$
\frac{1}{c^{\delta}} \leq \frac{f(c t)}{f(t)} \leq c \quad \text { for all } t>0, c \geq 1 \text { and some } 0<\delta<1 .
$$

Assume in addition that $\sqrt{f\left(x^{2}\right)}>x^{(n-d) / p}$ for large $x$ and suitable $0<d$ $\leq n$. Let $\Gamma$ be an $h$-set, where $h$ satisfies, for all $0<t, \lambda<1$,

$$
c_{1} \lambda^{s} \leq \frac{h(\lambda t)}{h(t)} \leq c_{2} \lambda^{d}
$$


for some $0<d \leq s \leq n$, and let $\mu$ be a fixed $h$-measure. Then there exists a time-changed Dirichlet form $(\check{\mathcal{E}}, \check{\mathcal{F}})$ with

$$
\begin{aligned}
\check{\mathcal{F}}=\mathcal{B}_{22}^{1, f}(\Gamma) & =\left\{u \in L_{2}(\Gamma):\right. \\
\quad \iint_{|x-y|<1} & \left.\frac{f\left(|x-y|^{-2}\right)}{|x-y|^{-n} h(|x-y|)^{2}}|u(x)-u(y)|^{2} \mu(d x) \mu(d y)<\infty\right\} .
\end{aligned}
$$

REMARK 28. Suppose there exists $\varepsilon>0$ such that $f(x) \leq x^{1-\varepsilon}$ as $x \rightarrow \infty$. The Dirichlet form $\left(\mathcal{E}^{\prime}, H^{f\left(|\cdot|^{2}\right), 1}\left(\mathbb{R}^{n}\right)\right)$, where

$$
\mathcal{E}^{\prime}(u, v)=\iint_{|x-y|<1} \frac{f\left(|x-y|^{-2}\right)}{|x-y|^{n}}(u(x)-u(y))(v(x)-v(y)) d x d y,
$$

is equivalent to $\left(\mathcal{E}, H^{f\left(|\cdot|^{2}\right), 1}\left(\mathbb{R}^{n}\right)\right)$, but cannot be obtained from it by some time change. Analogously, the Dirichlet form

$$
\begin{aligned}
& \widetilde{\mathcal{E}}(u, v) \\
& =\iint_{|x-y|<1} \frac{f\left(|x-y|^{-2}\right)}{|x-y|^{-n} h(|x-y|)^{2}}(u(x)-u(y))(v(x)-v(y)) \mu(d x) \mu(d y)
\end{aligned}
$$

is equivalent to $\check{\mathcal{E}}(\cdot, \cdot)$, but cannot be obtained from $\widetilde{\mathcal{E}}(\cdot, \cdot)$ by some time change. See [21] for a similar discussion.

Appendix I: Proof of Theorem 10. To prove the equivalence of Definitions 9 and 3 we need some technical statements. Now we will use a modification of the definition of $(\sigma, p)_{K}$-N-atoms, namely, we assume that they are located at $B\left(x^{k m}, c_{2} / N_{k}\right), c_{2}=c N_{r}$.

Lemma 29. The series $g=\sum_{\beta \in \mathbb{N}_{0}^{n}} g^{\beta}$, where

$$
g^{\beta}(x)=\sum_{k=0}^{\infty} \sum_{m \in \mathbb{Z}^{n}} \lambda_{k m}^{\beta}(\beta q u)_{k m}^{\sigma, N}(x),
$$

converges in $L_{p}\left(\mathbb{R}^{n}\right)$ for $1 \leq p<\infty$.

Proof. For an increasing admissible sequence $\left(\sigma_{j}\right)_{j \geq 0}$ there exists a small $\delta>0$ such that

$$
\sigma_{j}^{-1} \leq 2^{-j \delta}
$$

Then from (10) we have

$$
|g(x)| \leq C \sum_{\beta \in \mathbb{N}_{0}^{n}} \sum_{k=0}^{\infty} \sum_{m \in \mathbb{Z}^{n}} N_{r}^{|\beta|}\left|\lambda_{k m}^{\beta}\right| 2^{-\delta k} N_{k}^{n / p} 1_{k m}(x),
$$


where $1_{k m}(x)$ is now the characteristic function of the ball centered at $x^{k m}$ with radius $c_{2} / N_{k}$. Then

$$
|g(x)|^{p} \leq C \sum_{\beta \in \mathbb{N}_{0}^{n}} \sum_{k=0}^{\infty} \sum_{m \in \mathbb{Z}^{n}} N_{r+\varepsilon}^{|\beta| p}\left|\lambda_{k m}^{\beta}\right|^{p}\left(\frac{N_{r}}{N_{r+\varepsilon}}\right)^{|\beta| p} 2^{-\delta k} N_{k}^{n} 1_{k m}(x) .
$$

By the Hölder inequality we obtain

$$
\left\|g\left|L_{p}\left(\mathbb{R}^{n}\right)\left\|\leq C_{1}\right\| \lambda\right| b_{p p}\right\|_{\kappa}
$$

for some $\kappa>r$, and since $b_{p q} \subset b_{p p}$, we have

$$
\left\|g\left|L_{p}\left(\mathbb{R}^{n}\right)\left\|\leq C_{2}\right\| \lambda\right| b_{p q}\right\|_{\kappa}
$$

Lemma 30. Let $(\beta q u)_{k m}^{\sigma, N}$ be as in Definition 8. Then there exists a constant $C>0$ such that $C^{-1}(\beta q u)_{k m}^{\sigma, N}$ are $(\sigma, p)_{K}-N$-atoms located at $B\left(x^{k m}, c_{2} / N_{k}\right)$, and

$$
C<C_{1}(1+|\beta|)^{K} N_{r}^{|\beta|}
$$

where $C_{1}>0$ does not depend on $\beta$, and $r$ is from Definition 7 , that is, $c_{2}=c N_{r}$.

Proof. Let $K>0$ and $|\alpha| \leq K$. Then, assuming that $D^{\gamma}\left(x-x^{k m}\right)^{\beta}=0$ if $|\gamma|>|\beta|$, we have

$$
\begin{aligned}
\left|D^{\alpha}\left(x-x^{k m}\right)^{\beta} \theta^{k m}(x)\right| & \leq \sum_{|\gamma| \leq|\alpha|}\left|D^{\gamma}\left(x-x^{k m}\right)^{\beta} D^{\gamma} \theta^{k m}(x)\right| \\
& \leq c_{\beta} \sum_{|\gamma| \leq|\alpha|}\left(\frac{c N_{r}}{N_{k}}\right)^{|\beta-\gamma|}\left|D^{\gamma}\left(x-x^{k m}\right)^{\beta}\left(\frac{N_{k}}{c N_{r}}\right)^{|\beta-\gamma|}\right| N_{k}^{|\gamma|} \\
& \leq c_{\beta} N_{k}^{|\alpha|}\left(\frac{N_{r}}{N_{k}}\right)^{|\beta|}, \quad \text { where } c_{\beta} \leq C_{0}(1+|\beta|)^{\alpha} .
\end{aligned}
$$

Then we have the statement of our lemma with $C \leq C_{1}(1+|\beta|)^{K} N_{r}^{|\beta|}$.

Now we are ready to prove the equivalence theorem.

Proof of Theorem 10. We will follow the proof of Theorem 2.9 from [31]. Let

$$
g(x)=\sum_{\beta \in \mathbb{N}_{0}^{n}} \sum_{k=0}^{\infty} \sum_{m \in \mathbb{Z}^{n}} \lambda_{k m}^{\beta}(\beta q u)_{k m}^{\sigma, N}(x) \quad \text { in } S^{\prime}\left(\mathbb{R}^{n}\right)
$$

and

$$
\left\|\lambda\left|f_{p q}^{N}\left\|_{\varrho}=\sup _{\beta \in \mathbb{N}_{0}^{n}} N_{\varrho}^{|\beta|}\right\| \lambda^{\beta}\right| f_{p q}\right\|<\infty
$$


Let $g(x)=\sum_{\beta \in \mathbb{N}_{0}^{n}} g^{\beta}(x)$, where

$$
g^{\beta}(x)=\sum_{k=0}^{\infty} \sum_{m \in \mathbb{Z}^{n}} \lambda_{k m}^{\beta}(\beta q u)_{k m}^{\sigma, N}(x) .
$$

By Lemma 29, this Fourier series converges in $L_{p}\left(\mathbb{R}^{n}\right)$. Let now

$$
a_{k m}^{\beta}(x)=C^{-1}(\beta q u)_{k m}^{\sigma, N}(x),
$$

where $C^{-1}$ is from Lemma 30. Then $\left\{a_{k m}^{\beta}\right\}$ are $(\sigma, p)_{K}-N$-atoms for some large $K$, and thus

$$
g^{\beta}(x)=\sum_{k=0}^{\infty} \sum_{m \in \mathbb{Z}^{n}} \lambda_{k m}^{\beta} a_{k m}^{\beta}(x) .
$$

By the atomic decomposition theorem we have

$$
\left\|g^{\beta}\left|F_{p q}^{\sigma, N}\left(\mathbb{R}^{n}\right)\|\leq C\| \lambda^{\beta}\right| f_{p q}^{N}\right\|
$$

and by the triangle inequality and Lemma 30 we obtain

$$
\begin{aligned}
\left\|g \mid F_{p q}^{\sigma, N}\left(\mathbb{R}^{n}\right)\right\| & \leq \sum_{\beta \in \mathbb{N}_{0}^{n}}\left\|g^{\beta} \mid F_{p q}^{\sigma, N}\left(\mathbb{R}^{n}\right)\right\| \\
& \leq c \sum_{\beta \in \mathbb{N}_{0}^{n}}(1+|\beta|)^{K}\left(\frac{N_{r}}{N_{\varrho}}\right)^{|\beta|} N_{\varrho}^{|\beta|}\left\|\lambda^{\beta}\left|f_{p q}^{N}\left\|\leq c^{\prime}\right\| \lambda^{\beta}\right| f_{p q}^{N}\right\|_{\varrho},
\end{aligned}
$$

where $\varrho>r$. Thus $g \in F_{p q}^{\sigma, N}\left(\mathbb{R}^{n}\right)$.

Conversely, let $g \in F_{p q}^{\sigma, N}\left(\mathbb{R}^{n}\right)$, and consider the resolution of unity $\left\{\varphi_{k}\right\}$ as in Definition 2. Then

$$
\widehat{g}(\xi)=\sum_{k=0}^{\infty} \varphi_{k}(\xi) \widehat{g}(\xi), \quad \xi \in \mathbb{R}^{n}
$$

where the convergence is in $S^{\prime}\left(\mathbb{R}^{n}\right)$.

Let $Q_{k}$ be the cube in $\mathbb{R}^{n}$ centered at the origin and with side length $2 \pi N_{k}$. For simplicity we assume (because due to condition (3) the sequence $\left(N_{k}\right)_{k \in \mathbb{N}}$ is admissible) that $N_{k+1} \leq 2 \pi N_{k}$. Then $\operatorname{supp} \varphi_{k} \subset Q_{k}$. We expand $\varphi_{k} \widehat{g}$ in $Q_{k}$ by

$$
\left(\varphi_{k} \widehat{g}\right)(\xi)=\sum_{m \in \mathbb{Z}^{n}} b_{k m} e^{-i m N_{k}^{-1} \xi}, \quad \xi \in Q_{k}
$$

with

$$
b_{k m}=c N_{k}^{-n} \int_{Q_{k}} e^{i m N_{k}^{-1} \xi}\left(\varphi_{k} \widehat{g}\right)(\xi) d \xi=c^{\prime} N_{k}^{-n}\left(\varphi_{k} \widehat{g}\right)\left(m / N_{k}\right) .
$$

Let now $\Lambda=\left\{\Lambda_{k m}\right\}, k \in \mathbb{N}_{0}, m \in \mathbb{Z}^{n}$,

$$
\Lambda_{k m}=\sigma_{k} N_{k}^{-n / p}\left(\varphi_{k} \widehat{g}\right)\left(m / N_{k}\right)
$$


Then (see [30], [31], and [3])

$$
\left\|g\left|F_{p q}^{\sigma, N}\left(\mathbb{R}^{n}\right)\|\sim\| \Lambda\right| f_{p q}^{N}\right\| .
$$

Let $\phi \in S\left(\mathbb{R}^{n}\right)$ be a function such that, for $k \in \mathbb{N}_{0}, \phi_{k}=\phi\left(\xi / N_{k}\right)=1$ if $\xi \in \operatorname{supp} \varphi_{k}$ and $\operatorname{supp} \phi_{k} \subset Q_{k}$. Multiplying (51) by $\phi_{k}$ and extending by zero from $Q_{k}$ to $\mathbb{R}^{n}$, we get

$$
\begin{aligned}
\left(\phi_{k}\left(\varphi_{k} \widehat{g}\right)\right)^{\vee}(x) & =\sum_{m \in \mathbb{Z}^{n}} b_{k m} \phi_{k}^{\vee}\left(x-\frac{m}{N_{k}}\right)=N_{k}^{n} \sum_{m \in \mathbb{Z}^{n}} b_{k m} \phi_{k}^{\vee}\left(N_{k} x-m\right) \\
& =C \sum_{m \in \mathbb{Z}^{n}} \Lambda_{k m} \sigma_{k}^{-1} N_{k}^{n / p} \phi_{k}^{\vee}\left(N_{k} x-m\right), \quad x \in \mathbb{R}^{n} .
\end{aligned}
$$

By the Paley-Wiener-Schwartz Theorem we have, for some $b>0$,

$$
\left|D^{\alpha} \phi_{k}^{\vee}(x)\right| \leq c_{b} \alpha !(1+|x|)^{-b}, \quad x \in \mathbb{R}^{n} ;
$$

see $[31, \S 2.9]$.

Let $\left\{\theta^{k m}\right\}$ be as in Definition 7. We decompose the function $\phi_{k}^{\vee}\left(N_{k} x-m\right)$ with respect to the points $x^{k, l} / N_{\varrho}, \varrho>r$ :

$$
\phi_{k}^{\vee}\left(N_{k} x-m\right)=\sum_{\beta \in \mathbb{Z}^{n}} \frac{N_{k}^{|\beta|}}{\beta !} D^{\beta} \phi_{k}^{\vee}\left(\frac{x^{k, l} N_{k}}{N_{\varrho}}-m\right)\left(x-\frac{x^{k, l}}{N_{\varrho}}\right)^{\beta} .
$$

Therefore

$$
\begin{aligned}
\left(\phi_{k}\left(\varphi_{k} \widehat{g}\right)\right)^{\vee}(x)= & \sum_{m \in \mathbb{Z}^{n}} \sum_{l \in \mathbb{Z}^{n}} \sum_{\beta \in \mathbb{N}_{0}^{n}} \sigma_{k}^{-1} N_{k}^{n / p+|\beta|}\left(N_{\varrho} x-x^{k, l}\right)^{\beta} \theta^{k, l}\left(N_{\varrho} x\right) \\
& \times \frac{N_{\varrho}^{-|\beta|}}{\beta !} \Lambda_{k m} D^{\beta} \phi_{k}^{\vee}\left(\frac{x^{k, l} N_{k}}{N_{\varrho}}-m\right) \\
= & \sum_{m \in \mathbb{Z}^{n}} \sum_{l \in \mathbb{Z}^{n}} \sum_{\beta \in \mathbb{N}_{0}^{n}}(\beta q u)_{k, l}^{\sigma, N}\left(N_{\varrho} x\right) \lambda_{k, l}^{\beta},
\end{aligned}
$$

and from (52) we have

$$
\left|D^{\beta} \phi_{k}^{\vee}\left(\frac{x^{k, l} N_{k}}{N_{\varrho}}-m\right)\right| \leq \frac{c_{b}}{\beta !} \frac{1}{\left(1+\left|x^{k, l} N_{k} / N_{\varrho}-m\right|\right)^{b}} .
$$

Due to the structure of the approximate lattice (condition (8), the points $x^{k, l}$ are "close" to the point $\left.l \in \mathbb{Z}^{n}\right)$ there is $C_{1}>0$ such that $\left|x^{k, l} N_{k} / N_{\varrho}-m\right| \geq$ $C_{1}|l-m|$. Then

which leads to

$$
\left|D^{\beta} \phi_{k}^{\vee}\left(\frac{x^{k, l} N_{k}}{N_{\varrho}}-m\right)\right| \leq \frac{C_{2} \beta !}{(1+|l-m|)^{b}},
$$

$$
\left|\lambda_{k, l}^{\beta}\right| \leq C_{2} N_{\varrho}^{-|\beta|} \sum_{m \in \mathbb{Z}^{n}} \frac{\Lambda_{k m}}{(1+|l-m|)^{b}} \leq C_{3} N_{\varrho}^{-|\beta|} \sum_{m \in \mathbb{Z}^{n}} \frac{\Lambda_{k m+l}}{(1+|m|)^{b}},
$$


and thus

$$
\left\|\lambda^{\beta}\left|f_{p q}^{N}\left\|_{\varrho} \leq c_{\varrho} \sum_{m \in \mathbb{Z}^{n}}\left(\frac{1+|m|^{a}}{1+|m|^{b}}\right)\right\| \Lambda\right| f_{p q}^{N}\right\| \leq c_{\varrho}\left\|g \mid F_{p q}^{\sigma, N}\left(\mathbb{R}^{n}\right)\right\| .
$$

\section{Appendix II: Proof of Theorem 16}

Proof. This is a modification of the proof of Theorem 2.4.1 of [29], so we just give a short outline, and refer for details to [29].

Let $u \in S\left(\mathbb{R}^{n}\right)$. Since $\varphi_{j}(\xi) \widehat{u}(\xi)$ is not necessarily in $S\left(\mathbb{R}^{n}\right)$, we need functions $M$ and $H$ to justify the convergence near and far from zero.

Let $\left\{\varrho_{k}\right\}$ be a resolution of unity from Definition 2 , and let $\varrho_{l}=0$ if $l<0$. Put

$$
\widetilde{\varrho_{j}}(x)=\left|N_{j}^{-1} x\right|^{\alpha_{1}} \varrho_{j}(x), \quad j \in \mathbb{N}_{0} .
$$

Then by (31) and the inequality

$$
N_{l+j} \leq 2^{l \kappa} N_{j}, \quad l, j \in \mathbb{N},
$$

which follows from (3), we get

$$
\begin{aligned}
& \left|\sum_{l=-\infty}^{K} 2^{j \alpha}\left(\varphi_{j} \varrho_{l+j} \widehat{u}(\cdot)\right)^{\vee}(x)\right| \\
& \quad \leq \sum_{l=-\infty}^{K} 2^{l\left(\alpha_{1} \kappa-\alpha\right)}\left|\left(\frac{\varphi_{j}(z)}{\left|N_{j}^{-1} z\right|^{\alpha_{1}}} 2^{\alpha(j+l)} \widetilde{\varrho}_{j+l}(z) \widehat{u}(\cdot)\right)^{\vee}(x)\right| .
\end{aligned}
$$

Since supp $\widetilde{\varrho}_{j+l} \subset\left\{\xi \in \mathbb{R}^{n}: N_{j+l-1} \leq|\xi| \leq N_{j+l+1}\right\}$, we can replace $\varphi_{j}(z)$ with $\varphi_{j}(z) M\left(c z / N_{j}\right)$, where $c<1 / 2^{\kappa(l+1)}$. Using (31) we obtain

$$
\left|\sum_{l=-\infty}^{K} 2^{j \alpha}\left(\varphi_{j} \varrho_{l+j} \widehat{u}\right)^{\vee}(x)\right| \leq \sum_{l=-\infty}^{K} 2^{l\left(\alpha_{1} \kappa-\alpha\right)} 2^{\alpha(j+l)}\left(\widetilde{\varrho}_{j+l}^{*} u\right)_{a}(x),
$$

where $\left(\varrho_{k}^{*} f\right)_{a}$ is a maximal function:

$$
\left(\varrho_{k}^{*} u\right)_{a}(x)=\sup _{y \in \mathbb{R}^{n}} \frac{\left|\varrho_{k}(D) u(x-y)\right|}{\left(1+N_{k}|y|\right)^{a}}
$$

see [29] for details. Since $\alpha_{1} \kappa>\alpha$ we obtain

$$
\begin{aligned}
\left\|\left(\sum_{j=0}^{\infty} \sum_{l=-\infty}^{K}\left|2^{j \alpha}\left(\varphi_{j} \varrho_{l+j} \widehat{u}\right)^{\vee}(\cdot)\right|^{q}\right)^{1 / q} \mid L_{p}\left(\mathbb{R}^{n}\right)\right\| \\
\left.\leq C_{1} \|\left(\sum_{j=0}^{\infty} 2^{j \alpha q}\left(\varrho_{j}^{*} u\right)_{a}^{q}(\cdot)\right)^{q}\right)^{1 / q} \mid L_{p}\left(\mathbb{R}^{n}\right) \| .
\end{aligned}
$$


Analogously, putting $\varrho_{j}^{\prime}(x)=\left|N_{j}^{-1} x\right|^{\alpha_{0}} \varrho_{j}(x)$ we get

$$
\begin{aligned}
& \left|\sum_{l=K+1}^{\infty} 2^{j \alpha}\left(\varphi_{j} \varrho_{l+j} \widehat{u}\right)^{\vee}(x)\right| \\
& \leq \sum_{l=K+1}^{\infty} 2^{l\left(\alpha_{0} \kappa-\alpha\right)}\left|\left(\frac{\varphi_{j}(z)}{\left|N_{j}^{-1} z\right|^{\alpha_{0}}} 2^{\alpha(j+l)} \varrho_{j+l}^{\prime}(z) \widehat{u}(z)\right)^{\vee}(x)\right| .
\end{aligned}
$$

Replacing $\varphi_{j}(z)$ with $\varphi_{j}(z) H\left(N_{j+l}^{-1} z\right)$ we obtain, using (32), (53) and $\alpha>\alpha_{0} \kappa$, the inequality

$$
\begin{aligned}
\|\left(\sum_{j=0}^{\infty} \sum_{l=K+1}^{\infty}\left|2^{j \alpha}\left(\varphi_{j} \varrho_{l+j} \widehat{u}\right)^{\vee}(\cdot)\right|^{q}\right)^{1 / q} & \mid L_{p}\left(\mathbb{R}^{n}\right) \| \\
& \leq C_{2} 2^{K\left(\alpha_{0} \kappa-\alpha\right)}\left\|u \mid F_{p q}^{\sigma, N}\left(\mathbb{R}^{n}\right)\right\| .
\end{aligned}
$$

Now we need to show the reverse inequality. Let $f \in F_{p q}^{\sigma, N}\left(\mathbb{R}^{n}\right)$. We show that $\left\|f \mid F_{p q}^{\sigma, N}\left(\mathbb{R}^{n}\right)\right\|$ can be estimated from above by the quasi-norm (33), which we will denote by $\|\cdot\|_{\varphi}$.

Let $\psi \in S\left(\mathbb{R}^{n}\right)$ with $\operatorname{supp} \psi \in\{x:|x| \leq 2\}$, and $\psi(x)=1$ for $|x| \leq 1$. Let $R>1$, and put

$$
w_{R, j}=1-\sum_{l=R+1}^{\infty}\left(\psi_{j+l}-\psi_{j+l-1}\right)=1-\sum_{l=R+1}^{\infty} \theta_{j+l}
$$

with $\psi_{l}(x)=\psi\left(N_{l}^{-1} x\right)$. Note that $\left\{\theta_{l}\right\}$ is a decomposition of unity from Definition 2, and $\operatorname{supp} w_{R, j} \subset B\left(0, N_{R+j}\right)$.

We obtain

$$
\left|\left(\varrho_{j} \widehat{u}\right)^{\vee}(x)\right|=\left|\left(\varrho_{j} w_{R, j} \widehat{u}\right)^{\vee}(x)\right| \leq C_{3} \int_{\mathbb{R}^{n}}\left|\left(\varrho_{j} / \varphi_{j}\right)(y)\left(\varphi_{j} w_{R, j} \widehat{u}\right)^{\vee}(x-y)\right| d y .
$$

Then, analogously to step 5 in Theorem 2.4.1 from [29], we obtain, with $r<1$,

$$
\left|\left(\varrho_{j} \widehat{u}\right)^{\vee}(x)\right|^{r} \leq\left(N_{j+R} / N_{j}\right)^{n(1-r)} M\left|\left(\varphi_{j} w_{R, j} \widehat{u}\right)^{\vee}(x)\right|^{r},
$$

where $M(\cdot)$ is a Hardy-Littlewood maximal function. Since for the maximal functions for all $u \in L_{p}\left(l_{q}\right), u=\left(u_{k}\right)_{k \in \mathbb{N}_{0}}$, the inequality

$$
\left\|M u\left|L_{p}\left(l_{q}\right)\left\|\leq C_{4}\right\| u\right| L_{p}\left(l_{q}\right)\right\|, \quad 1<p<\infty, 1<q<\infty,
$$

holds (see [29, Theorem 2.2.2]), we see that

$$
\begin{aligned}
\left\|\left(\sum_{j=1}^{\infty}\left|2^{j \alpha}\left(\varrho_{j} \widehat{u}\right)^{\vee}(\cdot)\right|^{q}\right)^{1 / q} \mid L_{p}\left(\mathbb{R}^{n}\right)\right\|^{r} \\
\leq 2^{R \kappa n(1-r)}\left\|\left(\sum_{j=1}^{\infty}\left|2^{j \alpha}\left(\varphi_{j} w_{R, j} \widehat{u}\right)^{\vee}(\cdot)\right|^{q}\right)^{1 / q} \mid L_{p}\left(\mathbb{R}^{n}\right)\right\|^{r}
\end{aligned}
$$




$$
\begin{aligned}
\leq & 2^{R \kappa n(1-r)}\left(\left\|\left(\sum_{j=1}^{\infty}\left|2^{j \alpha}\left(\varphi_{j} \widehat{u}\right)^{\vee}(\cdot)\right|^{q}\right)^{1 / q} \mid L_{p}\left(\mathbb{R}^{n}\right)\right\|^{r}\right. \\
& \left.+\left\|\left(\sum_{j=1}^{\infty} \sum_{l=K+1}^{\infty}\left|2^{j \alpha}\left(\varphi_{j} \theta_{j+l} \widehat{u}\right)^{\vee}(\cdot)\right|^{q}\right)^{1 / q} \mid L_{p}\left(\mathbb{R}^{n}\right)\right\|^{r}\right) \\
\leq & 2^{R \kappa n(1-r)}\|u\|_{\varphi}+N_{R}^{n(1-r)-r\left(\alpha-\kappa \alpha_{0}\right)}\left\|u \mid F_{p q}^{\sigma, N}\left(\mathbb{R}^{n}\right)\right\|,
\end{aligned}
$$

where in the last step we used (54).

Choose $r$ such that $n(1-r)-r\left(\alpha-\kappa \alpha_{0}\right)<0$; then we may choose large $R$ so that the second term is less than $1 / 2\left\|u \mid F_{p q}^{\sigma, N}\left(\mathbb{R}^{n}\right)\right\|$. Thus we arrive at $\left\|u \mid F_{p q}^{\sigma, N}\left(\mathbb{R}^{n}\right)\right\| \leq C_{5}\|u\|_{\varphi}$.

The equivalence of norms (33) and (34) follows by the same arguments as in the proof of Theorem 2.4.1 from [29].

\section{Appendix III: Proof of Theorem 18}

Proof. As in [19], one can show, using (44), (29), the generalized HardyLittlewood inequality from [23], and replacing $2^{\nu \alpha}$ by $f\left(2^{2 j}\right)^{\alpha / 2}$ in the extension theorem from [19], that the extension operator $E$ given by (40) is continuous from $\mathcal{B}_{p p}^{\alpha, f}(\Gamma)$ to $B_{p p}^{\sigma, N}\left(\mathbb{R}^{n}\right)$, where for the former space we use the equivalent norm (38).

Now we show that $R E=I$.

Let $\triangle_{\nu}$ denote a set of cubes with side length $2^{-\nu}$. As in [19] and [20], we have

$$
\int_{t \in \triangle_{\nu}}\left|E u(t)-u\left(t_{0}\right)\right|^{p} d x \leq 2^{-\nu n} \int_{\left|t-t_{0}\right|<r+c 2^{-\nu}} \frac{\left|u(t)-u\left(t_{0}\right)\right|^{p}}{\mu\left(B\left(t, 2^{-\nu}\right)\right)} \mu(d t) .
$$

Let $\tau$ be such that $2^{-\tau}<r / \sqrt{n}<2^{-(\tau-1)}$. Then

$$
\begin{aligned}
\frac{1}{m\left(B\left(t_{0}, r\right)\right)} \int_{\left|t-t_{0}\right|<r} \mid E u(t) & -\left.u\left(t_{0}\right)\right|^{p} d t \\
& \leq h\left(2^{-\tau}\right) g_{\tau}\left(t_{0}\right)=h\left(2^{-\tau}\right) 2^{\tau \varepsilon} g_{\tau}\left(t_{0}\right) 2^{-\tau \varepsilon},
\end{aligned}
$$

where

$$
g_{\tau}\left(t_{0}\right)=\int_{\left|t-t_{0}\right|<c 2^{-\tau}} \frac{\left|u(t)-u\left(t_{0}\right)\right|}{m\left(B\left(t_{0}, 2^{-\tau}\right)\right) m\left(B\left(t, 2^{-\nu}\right)\right)} \mu(d t) .
$$

By the definition of the space $\mathcal{B}_{p p}^{\alpha, f}(\Gamma)$ we have

$$
\sum_{\tau=0}^{\infty} f\left(2^{2 \tau}\right)^{\alpha p / 2} 2^{-n \tau} \int g_{\tau}\left(t_{0}\right) \mu\left(d t_{0}\right)<\infty,
$$

and then

$$
\int g_{\tau}\left(t_{0}\right) \mu\left(d t_{0}\right)<f\left(2^{2 \tau}\right)^{-\alpha p / 2} 2^{n \tau} .
$$


Thus

$$
\sum_{\tau=0}^{\infty} \int h\left(2^{-\tau}\right) 2^{\tau \varepsilon} g_{\tau}\left(t_{0}\right) \mu\left(d t_{0}\right)<\sum_{\tau=0}^{\infty} f\left(2^{2 \tau}\right)^{-\alpha p / 2} 2^{\tau(n+\varepsilon)} h\left(2^{-\tau}\right),
$$

which is convergent if (29) holds. Then $h\left(2^{-\tau}\right) 2^{\tau \varepsilon} g_{\tau}\left(t_{0}\right)$ is uniformly bounded in $\tau$, and letting $\tau \rightarrow \infty$ in (55), we obtain

$$
\frac{1}{m\left(B\left(t_{0}, r\right)\right)} \int_{\left|t-t_{0}\right|<r}\left|E u(x)-u\left(t_{0}\right)\right|^{p} d x \rightarrow 0 \quad \text { as } r \rightarrow 0 .
$$

Thus $R E=I$.

For the case of ordinary Besov spaces the proof of the continuity of the restriction relies essentially on the estimates for the Bessel kernel

$$
G_{\alpha}(x)=\left(\frac{1}{\left(1+|\cdot|^{2}\right)^{\alpha / 2}}\right)^{\vee}(x) .
$$

We will try to get similar estimates for the kernel

$$
K_{\alpha}^{f}(x)=\left(\frac{1}{\left(1+f\left(|\cdot|^{2}\right)\right)^{\alpha / 2}}\right)^{\vee}(x) ;
$$

then the proof from [19] will apply with necessary modifications.

Let $f$ satisfy (4). Then (see Remarks 34 and 35 in Appendix IV) we get

$$
\left|K_{\alpha}^{f}(x)\right| \leq \frac{C_{1}}{|x|^{n} f^{\alpha / 2}\left(1 /|x|^{2}\right)}, \quad\left|\left(K_{\alpha}^{f}(x)\right)_{x_{j}}^{\prime}\right| \leq \frac{C_{2}}{|x|^{n+1} f^{\alpha / 2}\left(1 /|x|^{2}\right)}
$$

for all $0<j<n$ and $x \in B(0, R)$, where $C_{1}, C_{2}$ are some constants, depending on $n$ and $\alpha$.

Now we indicate the changes in the restriction theorem from [19].

Let $\mu$ be an $h$-measure, and $L(x)=f^{-\alpha / 2}(1 / x)$, where $f$ is a Bernstein function satisfying (4) with $\delta$ such that $0<\alpha \delta<1$. Let $\theta>0$; if $x^{d+\varepsilon}<$ $\left(x^{\gamma} / L\left(x^{2}\right)\right)^{\theta}<x^{s-\varepsilon}$ for some small $\varepsilon>0$ and for all $x \geq 1$, then

$$
\begin{gathered}
\int_{|t-y|<r}\left(\frac{|t-y|^{-\gamma}}{L\left(1 /|t-y|^{2}\right)}\right)^{\theta} \frac{\mu(d r)}{\mu(B(y, r))} \leq\left(\frac{C_{3}}{r^{\gamma} L(1 / r)}\right)^{\theta}, \\
\int_{r \leq|t-y|<1}\left(\frac{|t-y|^{-\gamma}}{L\left(1 /|t-y|^{2}\right)}\right)^{\theta} \frac{\mu(d r)}{\mu(B(y, r))} \leq\left(\frac{C_{4}}{r^{\gamma} L(1 / r)}\right)^{\theta},
\end{gathered}
$$

for $t \in \mathbb{R}^{n}, r \leq 1$, and $0<d \leq s \leq n$.

Let $\theta>0$; then by the same arguments as in Lemma 4 from [19] we deduce from (56) that

$$
\iint_{G} \frac{\left|K_{\alpha}^{f}(x-t)-K_{\alpha}^{f}(y-t)\right|^{\theta} \mu(d x) \mu(d y)}{\mu(B(x, r)) \mu(B(y, r))} \leq C_{5}\left(\frac{1}{r^{n} f^{\alpha / 2}\left(1 / r^{2}\right)}\right)^{\theta}
$$


if $x^{n} / f^{\alpha / 2}\left(x^{2}\right) \leq x^{s / \theta-\varepsilon}$ for all $x \geq 1$ and some $\varepsilon>0$, and $G=\left\{(x, y) \in\left(\mathbb{R}^{n}\right)^{2}\right.$ : $|x-y|<r,|y-t|<2 r\}$. The same estimate holds if $x^{d / \theta-1+\varepsilon} \leq x^{n} / f^{\alpha / 2}\left(x^{2}\right)$ for all $x \geq 1$ and some $\varepsilon>0$, and $G=\left\{x, y \in \mathbb{R}^{n}:|x-y|<r,|y-t|>2 r\right\}$. Analogously,

$$
\int_{D}\left|K_{\alpha}^{f}(t+h)-K_{\alpha}^{f}(t)\right|^{\theta} d t \leq \frac{|h|^{n}}{\left(|h|^{n} f^{\alpha / 2}\left(1 /|h|^{2}\right)\right)^{\theta}},
$$

where $D=\left\{t \in \mathbb{R}^{n}:|t|<2|h|\right\}$ or $D=\left\{t \in \mathbb{R}^{n}:|t|>2|h|\right\}$. Then for $u=K_{\alpha}^{f} * \omega, \omega \in L_{p}\left(\mathbb{R}^{n}\right)$, we get

$$
\left(\iint_{|x-y|<r}|u(x)-u(y)|^{p} \frac{\mu(d x) \mu(d y)}{\mu(B(x, r)) \mu(B(y, r))}\right)^{1 / p} \leq C_{6} \frac{\left\|\omega \mid L_{p}\left(\mathbb{R}^{n}\right)\right\|}{r^{n} f^{\alpha / 2}\left(1 / r^{2}\right)},
$$

and also

$$
\left\|u\left|L_{p}(\Gamma)\left\|\leq C_{7}\right\| \omega\right| L_{p}\left(\mathbb{R}^{n}\right)\right\|
$$

see [19] for the detailed proof in the case $f^{\alpha / 2}\left(x^{2}\right)=x^{\alpha}$. Note that

$$
\left\|\omega\left|L_{p}\left(\mathbb{R}^{n}\right)\|\sim\| u\right| B_{p p}^{\sigma, N}\left(\mathbb{R}^{n}\right)\right\| .
$$

Let $A$ and $B$ be some Banach spaces, and define

$$
l_{p}^{\alpha, f}(A)=\left\{\xi: \xi=\left(\xi_{j}\right)_{j \geq 0}, \xi_{j} \in A\right.
$$

and

$$
\left.\left\|\xi \mid l_{p}^{\alpha, f}\right\|=\left(\sum_{j=0}^{\infty} f\left(2^{2 j}\right)^{\alpha p / 2}\left\|\xi_{j} \mid A\right\|^{p}\right)^{1 / p}<\infty\right\}
$$

$$
l_{p}^{\alpha}(B)=\left\{\xi: \xi=\left(\xi_{j}\right)_{j \geq 0}, \xi_{j} \in B,\left\|\xi \mid l_{p}^{\alpha}\right\|=\left(\sum_{j=0}^{\infty} 2^{j \alpha p}\left\|\xi_{j} \mid B\right\|^{p}\right)^{1 / p}<\infty\right\},
$$

with the appropriate modification for $p=\infty$.

Let $0<\alpha_{0}<\alpha<\alpha_{1}, 0<\theta<1, \alpha=(1-\theta) \alpha_{0}+\theta \alpha_{1}$, and $1<p<$ $\infty$. Taking the sequence $\left(f\left(2^{2 j}\right)^{1 / 2}\right)_{j \geq 0}$ instead of $\left(2^{j}\right)_{j \geq 0}$ in the proof of Theorem 1.18.2 from [27], we get

$$
\left(l_{\infty}^{\alpha_{0}, f}(A), l_{\infty}^{\alpha_{1}, f}(A)\right)_{\theta, p}=l_{p}^{\alpha, f}(A),
$$

which for the case $A=L_{p}(\Gamma \times \Gamma, \mu(d x) \times \mu(d y))$ can be reformulated as

$$
\left(\mathcal{B}_{\infty \infty}^{\alpha_{0}, f}(\Gamma), \mathcal{B}_{\infty \infty}^{\alpha_{1}, f}(\Gamma)\right)_{\theta, p}=\mathcal{B}_{p p}^{\alpha, f}(\Gamma) .
$$

Here $\left(X_{0}, X_{1}\right)_{\theta, p}$ is the space obtained by real interpolation of the spaces $X_{0}$ and $X_{1}$; see [27].

Analogously, for $B=L_{p}\left(\mathbb{R}^{n}\right)$ and $\xi_{j}=\varphi_{j}^{N}(D) u$, where $\varphi_{j}^{N}$ is from Definition 3, Theorem 1.18.2 from [27] implies

$$
\left(B_{p p}^{\sigma_{0}, N}\left(\mathbb{R}^{n}\right), B_{p p}^{\sigma_{1}, N}\left(\mathbb{R}^{n}\right)\right)_{\theta, p}=B_{p p}^{\sigma, N}\left(\mathbb{R}^{n}\right),
$$

where $\sigma_{0}=\left(2^{j \alpha_{0}}\right)_{j \geq 0}, \sigma_{1}=\left(2^{j \alpha_{1}}\right)_{j \geq 0}, \sigma=\left(2^{j \alpha}\right)_{j \geq 0}$. 
Let

$$
\left(T_{\nu} u\right)(x, y)=\left(\frac{|u(x)-u(y)|}{\mu\left(B\left(x, 2^{-\nu}\right)\right) \mu\left(B\left(x, 2^{-\nu}\right)\right)}\right)^{1 / p} \quad \text { if }|x-y|<2^{-\nu},
$$

and 0 otherwise. From (59) we derive that the operator $T=\left(T_{\nu}\right)_{\nu \geq 0}$ is bounded from $B_{p p}^{\sigma, N}\left(\mathbb{R}^{n}\right)$ to $l_{\infty}^{\alpha, f}(A)$, where $A=L_{p}(\Gamma \times \Gamma, \mu(d x) \times \mu(d y))$.

By interpolation arguments $T$ is bounded from $B_{p p}^{\sigma, N}\left(\mathbb{R}^{n}\right)$ to $l_{p}^{\alpha, f}(A)$, and

$$
\|R u\|^{\alpha, f} \leq c\left\|u \mid B_{p p}^{\sigma, N}\left(\mathbb{R}^{n}\right)\right\|
$$

Appendix IV: Kernel estimates. We will derive (56) using a Tauberian type theorem. We prove a lemma for so-called extended regularly varying functions $L$ (see [2]), and show how it applies in our situation.

Let

$$
L^{*}(\lambda)=\limsup _{t \rightarrow \infty} \frac{L(t \lambda)}{L(\lambda)}, \quad L_{*}(\lambda)=\liminf _{t \rightarrow \infty} \frac{L(t \lambda)}{L(\lambda)}, \quad \lambda>0 .
$$

Definition 31. A function $L$ is called extended regularly varying (ER) if it is positive, measurable, and

$$
\lambda^{d} \leq L_{*}(\lambda) \leq L^{*}(\lambda) \leq \lambda^{c} \quad \text { for all } \lambda \geq 1
$$

for some constants $c$ and $d$.

The function is called O-regularly varying (OR) if it is positive, measurable, and

$$
0<L_{*}(\lambda) \leq L^{*}(\lambda)<\infty \quad \text { for all } \lambda \geq 1 .
$$

We also quote some notions relevant to representations of radial positivedefinite functions; see [14] and [24].

Let $B(\xi)$ be a positive-definite function, $B(0)<\infty$. Then by the BochnerKhinchin theorem there exists a finite measure $F(d x)$ on $\mathbb{R}^{n}$ such that

$$
B(\xi)=\int_{\mathbb{R}^{n}} e^{i \xi x} F(d x) \text { and } F\left(\mathbb{R}^{n}\right)=B(0) .
$$

(We note here that probabilists define the Fourier transform of a function $u$ as $\widehat{u}(\xi)=\int_{\mathbb{R}^{n}} e^{i \xi x} u(x) d x$, and in this section we will use this notation.) If $B(\xi)$ is radial, i.e. $B(\xi)=: B_{n}(|\xi|)$, then it admits the following representation:

$$
B_{n}(r)=\int_{0}^{\infty} Y_{n}(r \lambda) G(d \lambda)
$$

where

$$
G(t)=\int_{\{\lambda:|\lambda|<t\}} F(d \lambda) \quad \text { with } \quad G\left(\mathbb{R}_{+}^{1}\right)=F\left(\mathbb{R}^{n}\right)=B(0)<\infty,
$$


and

$$
Y_{n}(t)=2^{(n-2) / 2} \Gamma(n / 2) J_{(n-2) / 2}(t) t^{(2-n) / 2}
$$

is the spherical Bessel function, with

$$
J_{\nu}(t)=\sum_{m=0}^{\infty}(-1)^{m}(t / 2)^{2 m+\nu}(m ! \Gamma(m+\nu+1))^{-1}
$$

being the Bessel function of the first kind of order $\nu$ (see [1]). Now we state a Tauberian type theorem for $B_{n}(r)$.

LEMMA 32. Let $L$ be an ER function which satisfies

$$
\frac{1}{t^{\alpha}}<\frac{L(t \lambda)}{L(\lambda)}<t^{\alpha \delta}
$$

for all $\lambda>0, t>1$ and for some $0<\alpha<(n-3) / 2, n \geq 4,0<\delta \alpha<1$. The following statements are equivalent:

(a) $G(\lambda) \sim L(1 / \lambda)$ as $\lambda \rightarrow 0$;

(b) $B_{n}(r) \sim L(r)$ as $r \rightarrow \infty$.

Proof. $(\mathrm{a}) \Rightarrow(\mathrm{b})$. Let $r$ be large. We use the representation (64) of $B_{n}(r)$. Integrating (64) by parts, we get

$$
B_{n}(r)=2^{(n-2) / 2} \Gamma(n / 2) \int_{0}^{\infty} J_{n / 2}(t) t^{-n / 2+1} G(t / r) d t .
$$

Using (66) we get, for $0<\alpha<(n-3) / 2,0<\alpha \delta<1$,

$$
\frac{B_{n}(r)}{L(r)} \leq c_{n, 1}\left(\int_{0}^{1} J_{n / 2}(t) t^{-n / 2+1-\alpha \delta} d t+\int_{1}^{\infty} J_{n / 2}(t) t^{-n / 2+1+\alpha} d t\right)=c_{n, 2}
$$

and

$$
\frac{B_{n}(r)}{L(r)} \geq c_{n, 3}\left(\int_{0}^{1} J_{n / 2}(t) t^{-n / 2+1+\alpha} d t+\int_{1}^{\infty} J_{n / 2}(t) t^{-n / 2+1-\alpha \delta} d t\right)=c_{n, 4},
$$

where we used the fact that

$J_{n / 2}(t) t^{-n / 2+\sigma} \sim t^{\sigma} \quad$ as $t \rightarrow 0, \quad J_{n / 2}(t) t^{-n / 2+\sigma} \sim t^{-(n+1) / 2+\sigma} \quad$ as $t \rightarrow \infty$ (see [1]). Thus $B_{n}(r) \sim L(r)$ as $r \rightarrow \infty$.

$(\mathrm{b}) \Rightarrow(\mathrm{a})$. Let $\widehat{G}(y)=\int_{0}^{\infty} e^{i \lambda y} d G(\lambda)$. Then (see [24, Lemma 1.4.11])

$$
\widehat{G}(i y)=A(n) y \int_{0}^{\infty} \frac{u^{n-1}}{\left(u^{2}+y^{2}\right)^{(n+1) / 2}} B_{n}(u) d u .
$$


Hence, for large $y$ and for $0<\alpha<(n-3) / 2,0<\alpha \delta<1$ we obtain

$$
\begin{aligned}
\frac{\widehat{G}(i y)}{L(y)} & =c_{n, 5} \int_{0}^{\infty} \frac{t^{n-1}}{\left(1+t^{2}\right)^{(n+1) / 2}} \frac{B_{n}(t y)}{L(y)} d t \\
& \geq c_{n, 6}\left(\int_{0}^{1} \frac{t^{n-1+\alpha \delta}}{\left(1+t^{2}\right)^{(n+1) / 2}} d t+\int_{1}^{\infty} \frac{t^{n-1-\alpha}}{\left(1+t^{2}\right)^{(n+1) / 2}} d t\right)=c_{n, 7} .
\end{aligned}
$$

On the other hand,

$$
\frac{\widehat{G}(i y)}{L(y)} \leq c_{n, 8}\left(\int_{0}^{1} \frac{t^{n-1-\alpha}}{\left(1+t^{2}\right)^{(n+1) / 2}} d t+\int_{1}^{\infty} \frac{t^{n-1+\alpha \delta}}{\left(1+t^{2}\right)^{(n+1) / 2}} d t\right)=c_{n, 9},
$$

i.e. $\widehat{G}(i y) \sim L(y)$ as $y \rightarrow \infty$. Considering $\widehat{G}(i y)$ as the Laplace-Stieltjes transform of $G(\lambda)$, from [2, Theorem 2.10.2] we deduce that $G(\lambda) \sim L(1 / \lambda)$ as $\lambda \rightarrow 0$.

REMARK 33. The proof of (b) $\Rightarrow$ (a) holds for all $n \geq 1$ and $0<\alpha<n$.

Assume that $F(d x)$ admits the spectral density $\phi(x)$; then it is easy to see that $\phi(x)$ is also radial, and we put $\phi_{n}(|x|):=\phi(x)$. Making the variable change, we get

$$
G(\lambda)=\left|S_{n-1}(1)\right| \int_{0}^{\lambda} \tau^{n-1} \phi_{n}(\tau) d \tau
$$

where $\left|S_{n-1}(r)\right|$ is the surface area of the sphere $S_{n-1}(r)$ of radius $r$ in $\mathbb{R}^{n}$,

$$
\left|S_{n-1}(1)\right|=\frac{2 \pi^{n / 2}}{n \Gamma(n / 2)},
$$

and thus

$$
\phi_{n}(\lambda)=\frac{G^{\prime}(\lambda)}{\left|S_{n-1}(1)\right| \lambda^{n-1}} .
$$

Let $G$ be twice continuously differentiable, and

$$
\left|G^{(k)}(\lambda)\right| \leq k ! G(\lambda) / \lambda^{k}, \quad k=1,2 .
$$

Then for some constant $c>0$ we have

$$
\left|\phi_{n}(\lambda)\right| \leq c G(\lambda) / \lambda^{n} .
$$

Now we apply Lemma 32 to get estimate (56).

REMARK 34. Let $0<\alpha<n$, and $f$ be a Bernstein function satisfying

$$
\frac{1}{c^{\delta}} \leq \frac{f(c \lambda)}{f(\lambda)} \leq c
$$

for all $\lambda>0, c \geq 1$, and some $\delta$ such that $0<\alpha \delta<1$. Let

$$
B(\xi)=\frac{1}{\left(1+f\left(|\xi|^{2}\right)\right)^{\alpha / 2}} .
$$


Define $B_{n}(|\xi|):=B(\xi)$. Then $L(\lambda)=1 / f^{\alpha / 2}\left(\lambda^{2}\right)$ is an ER function satisfying (66), and $B_{n}(\lambda) \sim L(\lambda)$ as $\lambda \rightarrow \infty$. Since $B_{n}(\lambda) \in C^{\infty}\left(\mathbb{R}_{+}\right)$, also $G(\lambda)$ belongs to $C^{\infty}\left(\mathbb{R}_{+}\right)$. By Lemma 32 and Theorem 2.10 .2 from [2] we get $G^{\prime}(\lambda) \sim \lambda^{-1} L(1 / \lambda)$ and $G^{\prime \prime}(\lambda) \sim \lambda^{-2} L(1 / \lambda)$ as $\lambda \rightarrow 0$.

Then from (69) we derive for $K_{\alpha}^{f}(x)=\phi_{n}(|x|)=B(|\cdot|)^{\vee}(x), x \in B_{R}(0)$ (the ball of radius $R$ with center in 0 ), the estimate

$$
\left|K_{\alpha}^{f}(x)\right| \leq \frac{C_{1}}{|x|^{n} f^{\alpha / 2}\left(1 /|x|^{2}\right)} .
$$

Remark 35. From (67) we get

$$
\left(K_{\alpha}^{f}\right)_{x_{i}}^{\prime}(x)=\frac{1}{\left|S_{n-1}(1)\right|}\left(\frac{G^{\prime \prime}(|x|)}{|x|^{n-1}} \frac{x_{i}}{|x|}+\frac{G^{\prime}(|x|)}{|x|^{n+1}} \frac{x_{i}}{|x|}\right),
$$

and in view of $(68)$ we obtain

$$
\left|\left(K_{\alpha}^{f}\right)_{x_{i}}^{\prime}(x)\right| \leq \frac{C_{2}}{|x|^{n+1} f^{\alpha / 2}\left(1 /|x|^{2}\right)}, \quad i=1, \ldots, n .
$$

\section{References}

[1] H. Bateman and A. Erdélyi, Higher Transcendental Functions, in 3 volumes, Nauka, Moscow, 1966-1967 (in Russian).

[2] N. Bingham, C. Goldie and J. Teugels, Regular Variation, Cambridge Univ. Press, Cambridge, 1987.

[3] M. Bricchi, Tailored function spaces and related h-sets, PhD thesis, Univ. of Jena, 2002 .

[4] - Tailored Besov spaces and h-sets, Math. Nachr. 263-264 (2004), 36-52.

[5] D. Edmunds and H. Triebel, Spectral theory for isotropic fractal drums, C. R. Acad. Sci. Paris Sér. I Math. 326 (1998), 1269-1274.

[6] —, - Eigenfrequencies of isotropic fractal drums, in: The Maz'ya Anniversary Collection, Vol. 2 (Rostock, 1998), Oper. Theory Adv. Appl. 110, Birkhäuser, Basel, 1999, 81-102.

[7] W. Farkas, Function spaces of generalized smoothness and pseudo-differential operators associated to a continuous negative definite function, Habilitationsschrift, München, 2003.

[8] W. Farkas, N. Jacob and R. Schilling, Function spaces related to continuous negative definite functions: $\psi$-Bessel potential spaces, Dissertationes Math. 393 (2001).

[9] W. Farkas and H.-G. Leopold, Characterisations of function spaces of generalised smoothness, Ann. Mat. Pura Appl. 185 (2004), 1-62.

[10] M. Fukushima, Time changed Markov processes, Swansea lecture notes, 2003.

[11] M. Fukushima, Y. Ōshima and M. Takeda, Dirichlet Forms and Symmetric Markov Processes, de Gruyter, Berlin, 1994.

[12] W. Hansen and M. Zähle, Restricting isotropic $\alpha$-stable Lévy processes from $\mathbb{R}^{n}$ to fractal sets, Forum Math., to appear.

[13] D. Haroske and S. Moura, Continuity envelopes of spaces of generalised smoothness, entropy and approximation numbers, J. Approx. Theory 128 (2004), 151-174. 
[14] A. Ivanov and N. Leonenko, Statistical Analysis of Random Fields, Kluwer, Dordrecht, 1989.

[15] N. Jacob, Pseudo-Differential Operators and Markov Processes. Vol. 1: Fourier Analysis and Semigroups, Imperial College Press, London, 2001.

[16] -, Pseudo-Differential Operators and Markov Processes. Vol. 2: Generators and Their Potential Theory, Imperial College Press, London, 2002.

[17] N. Jacob and R. Schilling, Function spaces as Dirichlet spaces (about a paper by Maz'ya and Nagel), Z. Anal. Anwend. 24 (2005), 3-28.

[18] - - - Towards an $L_{p}$-potential theory for sub-Markovian semigroups: kernels and capacities, Acta Math. Sinica, to appear.

[19] A. Jonsson, Besov spaces on closed subsets of $\mathbb{R}^{n}$, Trans. Amer. Math. Soc. 341 (1994), 355-370.

[20] A. Jonsson and H. Wallin, Function Spaces on Subsets of $\mathbb{R}^{n}$, Math. Rep. 2, Harwood, London, 1984.

[21] T. Kumagai, Some remarks for stable-like processes on fractals, in: Fractals in Graz 2001, Trends Math., Birkhäuser, Basel, 2003, 185-196.

[22] - Function spaces and stochastic processes on fractals, in: Fractal Geometry and Stochastics III, Progr. Probab. 57, Birkhäuser, Basel, 2004, 221-234.

[23] L. Leindler, Generalization of inequalities of Hardy and Littlewood, Acta Sci. Math. (Szeged) 31 (1970), 279-285.

[24] N. Leonenko, Limit Theorems for Random Fields with Singular Spectrum, Kluwer, Dordrecht, 1999.

[25] S. Moura, On some characterizations of Besov spaces of generalized smoothness, preprint 05-30, Coimbra Univ., 2005.

[26] A. Stós, Symmetric $\alpha$-stable processes on d-sets, Bull. Polish Acad. Sci. Math. 48 (2000), 237-245.

[27] H. Triebel, Interpolation Theory, Function Spaces, Differential Operators, NorthHolland, Amsterdam, 1978.

[28] —, Theory of Function Spaces, Birkhäuser, Basel, 1983.

[29] —, Theory of Function Spaces II, Birkhäuser, Basel, 1992.

[30] - Fractals and Spectra, Birkhäuser, Basel, 1997.

[31] —, The Structure of Functions, Birkhäuser, Basel, 2001.

[32] D. Yang and Y. Lin, Spaces of Lipschitz type on metric spaces and their applications, Proc. Edinb. Math. Soc. 47 (2004), 709-752.

Mathematical Institute

University of Jena

Ernst-Abbe-Platz 2

07743 Jena, Germany

E-mail: vic_knopova@yahoo.co.uk zaehle@minet.uni-jena.de

Received September 22, 2005

Revised version December 31, 2005 